\title{
On the Barban-Davenport-Halberstam theorem: XIII
}

by

\author{
C. Hooley (Cardiff)
}

1. Introduction. Being still involved with the moments

$$
G(x, k)=\sum_{\substack{0<a \leq k \\(a, k)=1}} E^{2}(x ; a, k)=\sum_{\substack{0<a \leq k \\(a, k)=1}}\left(\theta(x ; a, k)-\frac{x}{\phi(k)}\right)^{2}
$$

and

$$
S(x, Q)=\sum_{k \leq Q} G(x, k)
$$

where

$$
\theta(x ; a, k)=\sum_{\substack{p \leq x \\ p \equiv a, \bmod k}} \log p,
$$

we continue by investigating a topic that may be seen as a synthesis of those studied in I, II, and XII (as before, we refer to former papers with the present title by the Roman number indicating their position in the series, details of those cited being given in the list of references at the end). In the first two of those papers we were mainly interested in Barban-Montgomery asymptotic formulae (with remainder terms) of the type

$$
S(x, Q) \sim Q x \log Q
$$

that were valid unconditionally for

$$
x \log ^{-A} x<Q \leq x
$$

and also conditionally for

$$
x^{1 / 2+\varepsilon}<Q \leq x
$$

on the extended Riemann hypothesis, while in the last paper, improving on the work of Friedlander and Goldston [1], we shewed unconditionally that

$$
G(x, k)>\left(\frac{1}{2}-\varepsilon\right) x \log k \quad\left(x>x_{0}(\varepsilon)\right)
$$

2000 Mathematics Subject Classification: Primary 11N13. 
for

$$
x e^{-A_{1} \sqrt{\log x}}<k \leq x
$$

and that

$$
G(x, k)>\left(\frac{3}{2}-\frac{\log x}{\log k}-\varepsilon\right) x \log k
$$

on the same hypothesis as before when

$$
x^{2 / 3+\varepsilon}<k \leq x .
$$

We are thus prompted to study lower bounds for the sum $S(x, Q)$ and, in particular, to see whether we can improve Liu's result [8] to the effect that

$$
S(x, Q)>\left(\frac{1}{4}-\varepsilon\right) Q x \log Q
$$

for

$$
x \exp \left(-\log ^{3 / 5-\varepsilon} x\right)<Q \leq x,
$$

since the conditions governing results (3) and (6) above imply that the $1 / 4$ in (10) may be replaced by 1 and $1 / 2$ for the respective more limited ranges (4) and

$$
x e^{-A_{2} \sqrt{\log x}}<Q \leq x .
$$

Yet, before we describe what we shall obtain, we should mention that attempts to derive comparable results about useful upper bounds are currently doomed to failure in the light of informal comments attributed to Montgomery regarding the basic Barban-Davenport-Halberstam theorem. Indeed, in the spirit of Montgomery's observations, we must note that the validity of any bound $S(x, Q)<A_{3} Q x \log Q$ in a range wider than (4) would certainly imply a refinement of the relation

$$
E(x ; a, k)=O\left(\frac{x}{\log ^{A} x}\right)
$$

for all $k \leq x^{1 / 2}$, which phenomenon cannot be substantiated in our current state of knowledge concerning possible exceptional zeros of the Dirichlet's $L$-functions formed with real characters.

The purpose of the present article is first to prove unconditionally that

$$
S(x, Q)>(1-\varepsilon) Q x \log Q \quad\left(x>x_{0}(\varepsilon)\right)
$$

in Liu's range (11) and then to shew that

$$
S(x, Q)>(2-1 / \alpha-\varepsilon) Q x \log Q \quad\left(x>x_{0}(\varepsilon)\right)
$$

for $Q=x^{\alpha}$ and $1 / 2<\alpha \leq 1$ provided that the Riemann zeta function $\zeta(s)$ have no zeros in the half-plane $\sigma>3 / 4$. Thus the lower bound implied by (3) remains true in a range of $Q$ wider than (12), while the lower order of magnitude more weakly implied still holds for (5) under an hypothesis 
much less stringent than before. In method as much as in subject matter, the presentation embodies features common to I and XII, the basic technique of I being combined inter alia with the use of Friedlander's and Goldston's ([2] and [1]) surrogate prime number function $\Lambda_{R}(n)$ that appeared in XII. But the requisite properties of $\Lambda_{R}(n)$ that enter into the work are mostly different from those needed before and we must therefore begin by establishing two lemmata concerning them, one of which involves the application of contour integral methods in a slightly unfamiliar context. Also, in contrast with XII, it is necessary to bring in a method involving exponential sums and a maximal large sieve in order to stretch the range of validity of our conditional theorem down to $Q>x^{1 / 2+\varepsilon}$; this is the complementary aspect of a careful technique that allows us to replace the Riemann hypothesis by our assumption of a weaker version thereof.

2. Notation. The letters $a, d, k, l, n, q, \delta, \eta$ denote positive integers; $L$ is a non-zero integer; $b$ and $m$ are integers that are positive save in the statement and proof of Lemma $1 ; h$ is an integer; $p$ is a (positive) prime number; $x$ is a positive real variable that is to be regarded as tending to infinity.

The usual meaning was assigned to $\theta(x ; a, k)$ in the introduction, the customary notation $\theta(x)$ being used when $k=1$.

The symbols $A, A_{1}, A_{2}, \ldots$ denote positive absolute constants, while $C_{1}, C_{2}$, and $C_{3}>0$ are definite constants whose actual values are irrelevant to our investigation; $\varepsilon, \varepsilon_{1}, \varepsilon_{2}$ are (small) positive constants that are not necessarily the same at each occurrence; the constants implied by the $O$-notation are usually absolute except in circumstances where they obviously may depend on $\varepsilon$.

When defined, the (positive) highest common factor and least common multiple of integers $r, s$ are denoted by $(r, s)$ and $[r, s]$, respectively; $\mu(n)$ and $d(n)$ denote the Möbius function and the divisor function.

3. Lemmata on a surrogate prime number function. As foreshadowed in the introduction, some of the properties demanded here of a surrogate prime number function $L(n)$ go beyond those needed in XII and include two that are in no way implicit in the usual developments of sieve methods such as Selberg's. Of the two most obvious candidates

$$
\Lambda_{R}^{*}(n)=\sum_{\substack{d \mid n \\ d \leq R}} \mu(d) \log \frac{R}{d}
$$

and 


$$
\Lambda_{R}(n)=V(R) \Lambda_{R}^{\prime}(n)=V(R) \sum_{\substack{d \mid n \\ d \leq R}} \lambda_{d} \quad(\text { as in XI (14)) }
$$

for $L(n)$, the first is the best choice for one of the new features required but fails to be adequate for the other when the conditional part of the treatment is reached. We therefore introduce $\Lambda_{R}(n)$ straightway into the work, assuming where necessary any results stated in XII but noting at once that extra initial preparations are made almost inevitable by the fact that now $R^{2}$ may exceed the limit $x$ for $n$. Furthermore, lest there be any scope for misunderstanding, we should stress that, regardless of its provenance or application, the definition of $\lambda_{d}$ in $\Lambda_{R}(n)$ depends only on $d$ and $R$ in the manner indicated in Section 3 of XII.

Defined to be $1 / 2-u$ for the intervals $0 \leq u<1$ and $0<u \leq 1$, respectively, the familiar functions

$$
\psi(u)=[u]+1 / 2-u
$$

and $\psi^{-}(u)$ of period 1 play an important rôle in the conditional part of the treatment and have their entrance in our first lemma about $\Lambda_{R}(n)$. The subject of this, for

$$
(b, l)=b_{1}
$$

and

$$
l \leq R
$$

is the sum

$$
\Upsilon_{R}(v, w ; b, l)=\sum_{\substack{v<m \leq w \\ m \equiv b, \bmod l}} \Lambda_{R}(m)
$$

which by (13) equals

$$
V(R) \sum_{\substack{v<m \leq w \\ m \equiv b, \bmod l}} \sum_{\substack{d \mid m \\ d \leq R}} \lambda_{d}=V(R) \sum_{d \leq R} \lambda_{d} \sum_{\substack{v<m \leq w \\ m \equiv b, \bmod l \\ m \equiv 0, \bmod d}} 1 .
$$

Since the congruences in the last inner sum are compatible if and only if $(d, l) \mid b$ and hence if and only if $(d, l) \mid b_{1}$ by (15), we write

(19) $\quad l=\eta l^{\prime}, \quad d=\eta d^{\prime}, \quad m=\eta m^{\prime} \quad$ where $\quad \eta \mid b_{1} \quad$ and $\quad\left(l^{\prime}, d^{\prime}\right)=1$,

deducing that in this instance

$$
m^{\prime} \equiv b / \eta, \bmod l^{\prime} \quad \text { and } \quad m^{\prime} \equiv 0, \bmod d^{\prime} .
$$

The solutions of these, mod $l^{\prime} d^{\prime}$, being given by

$$
m^{\prime} \equiv(b / \eta) d^{\prime} \bar{d}^{\prime}, \bmod l^{\prime} d^{\prime},
$$


when $\bar{d}^{\prime}$ is defined, mod $l^{\prime}$, by

$$
d^{\prime} \bar{d}^{\prime} \equiv 1, \bmod l^{\prime},
$$

we end the first part of the estimation by inferring through (14) that the final inner sum in (18) is

$$
\begin{aligned}
& {\left[\frac{w / \eta-(b / \eta) d^{\prime} \bar{d}^{\prime}}{l^{\prime} d^{\prime}}\right]-\left[\frac{v / \eta-(b / \eta) d^{\prime} \bar{d}^{\prime}}{l^{\prime} d^{\prime}}\right]} \\
& =\frac{w-v}{\eta l^{\prime} d^{\prime}}+\psi\left(\frac{w / \eta-(b / \eta) d^{\prime} d^{\prime}}{l^{\prime} d^{\prime}}\right)-\psi\left(\frac{v / \eta-(b / \eta) d^{\prime} d^{\prime}}{l^{\prime} d^{\prime}}\right) .
\end{aligned}
$$

The influence on $\Upsilon_{R}(v, w ; b, l)$ of the first constituent on the second line of $(20)$ is seen by (18) to be

$$
\frac{V(R)(w-v)}{l} \sum_{\substack{d \leq R \\(d, l) \mid b_{1}}} \frac{\lambda_{d}}{d}(d, l)=\frac{(w-v)}{l} \Sigma_{A}, \quad \text { say, }
$$

to evaluate which we introduce the function

$$
\Phi_{a}(m)= \begin{cases}m & \text { if } m \mid a \\ 0 & \text { otherwise }\end{cases}
$$

that is to be expressed in the form

$$
\sum_{\delta \mid m} \Psi_{a}(\delta)
$$

Here, by the Möbius inversion formula,

$$
\Psi_{a}(\delta)=\sum_{\delta_{1} \mid \delta} \mu\left(\frac{\delta}{\delta_{1}}\right) \Phi_{a}\left(\delta_{1}\right)=\sum_{\delta_{1}\left|\delta ; \delta_{1}\right| a} \mu\left(\frac{\delta}{\delta_{1}}\right) \delta_{1}
$$

is Ramanujan's function $c_{\delta}(a)$, and we deduce that

$$
\begin{aligned}
\Sigma_{A} & =V(R) \sum_{d \leq R} \frac{\lambda_{d}}{d} \sum_{\delta|d ; \delta| l} c_{\delta}\left(b_{1}\right)=V(R) \sum_{\delta \mid l} c_{\delta}\left(b_{1}\right) \sum_{\substack{d \leq R \\
d \equiv 0, \bmod \delta}} \frac{\lambda_{d}}{d} \\
& =\sum_{\delta \mid l} \frac{c_{\delta}\left(b_{1}\right) \mu(\delta)}{\phi(\delta)}
\end{aligned}
$$

by (16) and the well-known formula

$$
\sum_{\substack{d \leq R \\ d \equiv 0, \bmod \delta}} \frac{\lambda_{d}}{d}=\frac{\mu(\delta)}{V(R) \phi(\delta)} \quad(\delta \leq R)
$$

expressed in XII (10). Thus, since $c_{p}\left(b_{1}\right)=-1$ or $p-1$ according as $p \nmid b_{1}$ 
or $p \mid b_{1}$,

$$
\Sigma_{A}=\prod_{p \mid l}\left(1-\frac{c_{p}\left(b_{1}\right)}{p-1}\right)=\frac{l}{\phi(l)} v\left(b_{1}\right)
$$

where

$$
v(a)= \begin{cases}1 & \text { if } a=1, \\ 0 & \text { if } a>1,\end{cases}
$$

the corresponding contribution to $\Upsilon_{R}(v, w ; b, l)$ being

$$
\frac{(w-v) v\left(b_{1}\right)}{\phi(l)}=\frac{(w-v) v\{(b, l)\}}{\phi(l)}
$$

by (21) and (15).

On the other hand, since

$$
V(R) \lambda_{d}=O\left(\log \frac{2 R}{d}\right)
$$

by XII (13), the contribution of the last two terms in $(20)$ to $\Upsilon_{R}(v, w ; b, l)$ is trivially

$$
O\left(\sum_{d \leq R} \log \frac{2 R}{d}\right)=O(R),
$$

which on being combined with (23) in (18) yields the first part of

Lemma 1. Let $\Upsilon_{R}(v, w ; b, l)$ and $v(a)$ be defined as in (17) and (22), respectively, and suppose that $l \leq R$. Then

$$
\Upsilon_{R}(v, w ; b, l)=\frac{(w-v) v\{(b, l)\}}{\phi(l)}+O(R) .
$$

Also, if $l^{\prime}$ denote $l / \eta$ for any given positive divisor $\eta$ of $l$, then the remainder term above can be replaced by the (explicit) expression

$$
\begin{aligned}
V(R) \sum_{\eta \mid(b, l)} & \sum_{\substack{d^{\prime} \leq R / \eta \\
\left(d^{\prime}, l^{\prime}\right)=1}} \lambda_{d^{\prime} \eta} \\
& \times\left\{\psi\left(\frac{w / \eta-(b / \eta) d^{\prime} \bar{d}^{\prime}}{l^{\prime} d^{\prime}}\right)-\psi\left(\frac{v / \eta-(b / \eta) d^{\prime} \bar{d}^{\prime}}{l^{\prime} d^{\prime}}\right)\right\} .
\end{aligned}
$$

Furthermore, there are parallel results for sums that only differ from $\Upsilon_{R}(v, w ; b, l)$ in the substitution of strict for unstrict or unstrict for strict inequalities in the definition of the range of summation of $m$; if, for example, the range of $m$ is $v<m<w$, then the first $\psi$ function in (25) must be replaced by $\psi^{-}$.

The second part, which has an obvious origin in (19) and (20), will be needed in preference to the first part when we come on to the conditional aspect of the treatment. A similar result is available for the corresponding 
sum formed with $\Lambda_{R}^{*}(n)$ instead of $\Lambda_{R}(n)$ save that one must include an extra remainder term arising from the summation of a series containing the Möbius function; this additional feature does not vitiate the unconditional aspect of the proof but totally compromises the other part of the treatment because even on the Riemann hypothesis the new arrival is too large.

The second lemma describes the behaviour for larger values of $R$ of the sum

$$
\Sigma_{x, R}=\sum_{n \leq x} \Lambda_{R}^{2}(n),
$$

for which Selberg's formula

$$
\Sigma_{x, R}=x V(R)+O\left(R^{2}\right)
$$

has already been stated in XII (15). But for $R>x^{1 / 2} \log ^{1 / 2} x$ this result becomes nugatory, a phenomenon that reflects the apparently growing disassociation of the underlying sieve process with the formal sum

$$
\sum_{d_{1}, d_{2} \leq R} \frac{\lambda_{d_{1}} \lambda_{d_{2}}}{\left[d_{1}, d_{2}\right]}=\frac{1}{V(R)}
$$

as $R$ increases toward $x$. Indeed, since the genesis of (26) is the transformation of $\Sigma_{x, R}$ into

$$
V^{2}(R) \sum_{d_{1}, d_{2} \leq R} \lambda_{d_{1}} \lambda_{d_{2}}\left[\frac{x}{\left[d_{1}, d_{2}\right]}\right]
$$

the most obvious portrayal of $\Sigma_{x, R}$ for $R \geq x^{1 / 2}$ would contain an explicit term

$$
x V^{2}(R) \sum_{\substack{d_{1}, d_{2} \leq R \\\left[d_{1}, d_{2}\right] \leq x}} \frac{\lambda_{d_{1}} \lambda_{d_{2}}}{\left[d_{1}, d_{2}\right]}
$$

in place of $x V(R)$ even though the former is not easy to estimate directly in terms of the already chosen values of $\lambda_{d}$. Also, while (28) is a conditionally non-negative quadratic form in $\lambda_{d}$ when $\lambda_{1}=1$, the same cannot necessarily be said of (29) in the absence of any prior knowledge of the difference between it and (28). No relief, therefore, can be expected from an attempt to adjust the values of $\lambda_{d}$ to those answering to a conditional state of (29), a realization that is strengthened by the fact that when $R=x$ the minimum of (28) becomes (the relatively very small) $V^{2}(R)$ for values $\lambda_{d}=\mu(d)$ that are a wholly unsuitable foundation for an ersatz von Mangoldt function.

We must therefore seek a more oblique method for estimating $\Sigma_{x, R}$ for larger values of $R$. Having rejected the idea of appealing to Graham's asymptotic formula [3]

$$
\sum_{n \leq x} \Lambda_{R}^{* 2}(n)=x \log R+O(x)
$$


because of an insufficiently close likeness between $\Lambda_{R}(n)$ and $\Lambda_{R}^{*}(n)$, we suppose that

$$
R \leq x
$$

and adopt a complex variable method in which the starting point is the study of the function $L_{R}(s)$ defined by the Dirichlet's series

$$
\sum_{n=1}^{\infty} \frac{\Lambda_{R}^{2}(n)}{n^{s}}
$$

for $\sigma>1$. This, much as in the development of Selberg's method, is by (13) equal to

$$
\begin{aligned}
& V^{2}(R) \sum_{n=1}^{\infty} \frac{1}{n^{s}} \sum_{\substack{d_{1}\left|n ; d_{2}\right| n \\
d_{1}, d_{2} \leq R}} \lambda_{d_{1}} \lambda_{d_{2}} \\
& =V^{2}(R) \sum_{d_{1}, d_{2} \leq R} \lambda_{d_{1}} \lambda_{d_{2}} \sum_{\substack{n=1 \\
n \equiv 0, \bmod \left[d_{1}, d_{2}\right]}}^{\infty} \frac{1}{n^{s}} \\
& =\zeta(s) V^{2}(R) \sum_{d_{1}, d_{2} \leq R} \frac{\lambda_{d_{1}} \lambda_{d_{2}}}{\left[d_{1}, d_{2}\right]^{s}}=\zeta(s) V^{2}(R) F_{R}(s), \quad \text { say, }
\end{aligned}
$$

which equation furnishes the analytic continuation of $L_{R}(s)$ over the entire plane. Also, for $\sigma<1,(24)$ implies that

$$
V^{2}(R) F_{R}(s)=O\left(\log ^{2} R \sum_{m \leq R^{2}} \frac{d_{3}(m)}{m^{\sigma}}\right)=O\left(\frac{R^{2(1-\sigma)} \log ^{4} R}{1-\sigma}\right)
$$

by partial summation, where here, as later, a little care must be taken because $\sigma$ may be close to 1 . Then, deciding to consider in the first place the sum

$$
\Sigma_{x, R}^{(1)}=\sum_{n \leq x}(x-n) \Lambda_{R}^{2}(n)
$$

instead of $\Sigma_{x, R}$ for ease of treatment, we have at once from (32) that

$$
\begin{aligned}
\Sigma_{x, R}^{(1)} & =\frac{1}{2 \pi i} \int_{c-i \infty}^{c+i \infty} L_{R}(s) \frac{x^{s+1}}{s(s+1)} d s \\
& =\frac{1}{2 \pi i} \int_{c-i \infty}^{c+i \infty} \zeta(s) V^{2}(R) F_{R}(s) \frac{x^{s+1}}{s(s+1)} d s
\end{aligned}
$$

for $c>1$ and, shifting the line of integration to $\sigma=1-\beta$ for a suitably 
small positive value of $\beta$, infer that

$$
\Sigma_{x, R}^{(1)}=\frac{1}{2} V^{2}(R) F_{R}(1) x^{2}+\frac{1}{2 \pi i} \int_{1-\beta-i \infty}^{1-\beta+i \infty} \zeta(s) V^{2}(R) F_{R}(s) \frac{x^{s}}{s(s+1)} d s
$$

because $\zeta(s)$ has a pole with residue 1 at $s=1$ and is

$$
O\left\{(|t|+1)^{1 / 8}\right\}
$$

along the second contour. Hence, by (32) and (27),

$$
\begin{aligned}
\Sigma_{x, R}^{(1)} & =\frac{1}{2} V(R) x^{2}+\frac{1}{2 \pi i} \int_{1-\beta-i \infty}^{1-\beta+i \infty} \zeta(s) V^{2}(R) F_{R}(s) \frac{x^{s}}{s(s+1)} d s \\
& =\frac{1}{2} V(R) x^{2}+I_{x, R}, \quad \text { say, }
\end{aligned}
$$

with which equation we end the initial and simpler part of the calculation.

To treat $I_{x, R}$ we shall choose $\beta$ and $T$ in terms of $x$ and $R$ according to the theory of $\zeta\left(s_{1}\right)=\zeta\left(\sigma_{1}+i t_{1}\right)$ in such a manner that $\left({ }^{1}\right)$

$$
\beta=A_{4} / \log T
$$

and that within the region

$$
\left|t_{1}\right| \leq 2 T, \sigma_{1} \geq 1-2 \beta,
$$

we have

(38) $\zeta\left(s_{1}\right)-\frac{1}{s_{1}-1}=O\left\{\log \left(\left|t_{1}\right|+2\right)\right\}=O(\log T), \quad \frac{1}{\zeta\left(s_{1}\right)}=O(\log T)$.

Then

$$
\begin{aligned}
I_{x, R} & =\frac{1}{2 \pi i}\left\{\int_{1-\beta-i T}^{1-\beta+i T}+\left(\int_{1-\beta-i \infty}^{1-\beta-i T}+\int_{1-\beta+i T}^{1-\beta+i \infty}\right)\right\} \zeta(s) V^{2}(R) F_{R}(s) \frac{x^{s+1}}{s(s+1)} d s \\
& =I_{x, R}^{(1)}+I_{x, R}^{(2)}, \quad \text { say, }
\end{aligned}
$$

wherein the second integral is dismissed at once by a crude argument involving (33) and (34). Indeed, because we see that

$$
I_{x, R}^{(2)}=O\left(\frac{x^{2}\left(R^{2} / x\right)^{\beta} \log ^{4} R}{\beta} \int_{T}^{\infty} \frac{d t}{t^{15 / 8}}\right)=O\left(\frac{x^{2}\left(R^{2} / x\right)^{\beta} \log ^{4} x}{T^{3 / 4}}\right)
$$

by (30) and (34), we are left with the term $I_{x, R}^{(1)}$ in

$$
I_{x, R}=I_{x, R}^{(1)}+O\left(\frac{x^{2}\left(R^{2} / x\right)^{\beta} \log ^{4} x}{T^{3 / 4}}\right)
$$

$\left({ }^{1}\right)$ The sharp form of the prime-number theorem cited later stems from an improvement of what is possible under (36), (37), and (38); such improvements here, however, have little influence on the quality of the lemma we are establishing. 
whose estimation involves the properties of $\zeta\left(s_{1}\right)$ connected with (36) and (37) above.

The most important element in the integrand of $I_{x, R}^{(1)}$ is $F_{R}(s)$, for which (33) must be superseded on the relevant contour

$$
\sigma=1-\beta, \quad|t| \leq T
$$

by an estimate founded on the equation

$$
\begin{aligned}
V^{2}(R) F_{R}(s) & =V^{2}(R) \sum_{d_{1}, d_{2} \leq R} \frac{\left\{\left(d_{1}, d_{2}\right)\right\}^{s} \lambda_{d_{1}} \lambda_{d_{2}}}{d_{1}^{s} d_{2}^{s}} \\
& =V^{2}(R) \sum_{d_{1}, d_{2} \leq R} \frac{\lambda_{d_{1}} \lambda_{d_{2}}}{d_{1}^{s} d_{2}^{s}} \sum_{\varrho\left|d_{1} ; \varrho\right| d_{2}} \phi(s, \varrho) \\
& =\sum_{\varrho \leq R} \mu^{2}(\varrho) \phi(s, \varrho)\left(V(R) \sum_{\substack{d \leq R \\
d \equiv 0, \bmod \varrho}} \frac{\lambda_{d}}{d^{s}}\right)^{2} \\
& =\sum_{\varrho \leq R} \frac{\mu^{2}(\varrho) \phi(s, \varrho)}{\varrho^{2 s}}\left(V(R) \sum_{\substack{d^{\prime} \leq R / \varrho \\
\left(d^{\prime}, \varrho\right)=1}} \frac{\lambda_{d^{\prime} \varrho}}{d^{\prime s}}\right) \\
& =\sum_{\varrho \leq R} \frac{\mu^{2}(\varrho) \phi(s, \varrho)}{\varrho^{2 s}} \Gamma^{2}(s, R, \varrho), \quad \text { say, }
\end{aligned}
$$

that is obtained in the customary way by setting

$$
\phi(s, \varrho)=\sum_{\Delta \mid \varrho} \mu\left(\frac{\varrho}{\Delta}\right) \Delta^{s}=\prod_{p \mid \varrho}\left(p^{s}-1\right)
$$

for square-free values of $\varrho$. Next, by the formula

$$
V(R) \lambda_{d}=\frac{\mu(d) d}{\phi(d)} \sum_{\substack{\varrho^{\prime} \leq R / d \\\left(\varrho^{\prime}, d\right)=1}} \frac{\mu^{2}\left(\varrho^{\prime}\right)}{\phi\left(\varrho^{\prime}\right)}
$$

given for example on p. 9 of our tract [4],

$$
\begin{aligned}
\Gamma(s, R, \varrho) & =\frac{\mu(\varrho) \varrho}{\phi(\varrho)} \sum_{\substack{d^{\prime} \leq R / \varrho \\
\left(d^{\prime}, \varrho\right)=1}} \frac{\mu\left(d^{\prime}\right) d^{\prime}}{\phi\left(d^{\prime}\right) d^{\prime s}} \sum_{\substack{\varrho^{\prime} \leq R /\left(d^{\prime} \varrho\right) \\
\left(\varrho^{\prime}, d^{\prime} \varrho\right)=1}} \frac{\mu^{2}\left(\varrho^{\prime}\right)}{\phi\left(\varrho^{\prime}\right)} \\
& =\frac{\mu(\varrho) \varrho}{\phi(\varrho)} \sum_{\substack{\varrho^{\prime} \leq R / \varrho \\
\left(\varrho^{\prime}, \varrho\right)=1}} \frac{\mu^{2}\left(\varrho^{\prime}\right)}{\phi\left(\varrho^{\prime}\right)} \sum_{\substack{d^{\prime} \leq R /\left(\varrho \varrho^{\prime}\right) \\
\left(d^{\prime}, \varrho \varrho^{\prime}\right)=1}} \frac{\mu\left(d^{\prime}\right) d^{\prime}}{\phi\left(d^{\prime}\right) d^{\prime s}},
\end{aligned}
$$


the inner sum being an example of the sums

$$
M_{k}(s, z)=\sum_{\substack{m \leq z \\(m, k)=1}} \frac{\mu(m) m}{\phi(m) m^{s}}
$$

that must now be estimated under the assumptions

$$
1 \leq z \leq R
$$

and (42).

First, by Euler's theorem, the Dirichlet's series

$$
G_{k}(w)=\sum_{\substack{m=1 \\(m, k)=1}}^{\infty} \frac{\mu(m) m}{\phi(m) m^{w}} \quad(w=v+\iota v)
$$

associated with $M_{k}(s)$ is equal to

$$
\begin{aligned}
\prod_{p \nmid k}( & \left.1-\frac{p}{(p-1) p^{w}}\right) \\
= & \prod_{p \mid k}\left\{1-\left(1-\frac{1}{p}\right)^{-1} \frac{1}{p^{w}}\right\}^{-1} \\
& \times \frac{1}{\zeta(w)} \prod_{p}\left\{\left(1-\left(1-\frac{1}{p}\right)^{-1} \frac{1}{p^{w}}\right)\left(1-\frac{1}{p^{w}}\right)^{-1}\right\} \\
= & \frac{1}{\zeta(w)} \prod_{p \mid k}\left\{1-\left(1-\frac{1}{p}\right)^{-1} \frac{1}{p^{w}}\right\} \prod_{p}\left\{1-\frac{1}{p^{w}(p-1)}\left(1-\frac{1}{p^{w}}\right)^{-1}\right\} \\
= & \frac{1}{\zeta(w)} H_{k}(w) B(w), \quad \text { say, }
\end{aligned}
$$

for $v>1$, where actually $B(w)$ is an absolutely bounded regular function and

$$
H_{k}(w)=O\left\{\sigma_{-3 / 4}(k)\right\}
$$

for $v>7 / 8$. Secondly, supposing initially that $z$ is the sum of $1 / 2$ and a positive integer $M$ and then applying Perron's formula to the function $G_{k}\left(s+s^{\prime}\right)$ quâ a Dirichlet's series in $s^{\prime}$, we have

$$
\begin{aligned}
M_{k}(s, z)= & \frac{1}{2 \pi i} \int_{2 \beta-i T}^{2 \beta+i T} \frac{1}{\zeta\left(s+s^{\prime}\right)} H_{k}\left(s+s^{\prime}\right) B\left(s+s^{\prime}\right) \frac{z^{s^{\prime}}}{s^{\prime}} d s^{\prime} \\
& +O\left(\frac{z^{2 \beta}}{T} \sum_{m=1}^{\infty} \frac{\mu^{2}(m)}{m^{\beta} \phi(m)|\log (z / m)|}\right)
\end{aligned}
$$




$$
\begin{aligned}
= & \frac{1}{\zeta(s)} H_{k}(s) B(s)+\frac{1}{2 \pi i}\left\{\int_{-\beta-i T}^{-\beta+i T}+\int_{-\beta+i T}^{2 \beta+i T}+\int_{-\beta-i T}^{2 \beta-i T}\right\} \\
& \times \frac{1}{\zeta\left(s+s^{\prime}\right)} H_{k}\left(s+s^{\prime}\right) B\left(s+s^{\prime}\right) \frac{z^{s^{\prime}}}{s^{\prime}} d s^{\prime}+O\left(\frac{z^{2 \beta}}{T} \Sigma_{B}\right), \quad \text { say },
\end{aligned}
$$

after we have moved the contour of integration leftward through the pole at $s^{\prime}=0$. The first term in this is

$$
O\left\{\sigma_{-3 / 4}(k) \log T\right\}
$$

by (38), (48), and assumption (42); similarly, since $s_{1}=s+s^{\prime}$ in the following integrals adheres to the conditions $\left|t+t^{\prime}\right| \leq 2 T, \sigma+\sigma^{\prime} \geq 1-2 \beta$ corresponding to (37), the sum of these integrals is

$$
\begin{aligned}
O\left(z^{-\beta} \sigma_{-3 / 4}(k) \log T \int_{0}^{T} \frac{d t}{\beta+t}\right)+O\left(\frac{\beta z^{2 \beta} \sigma_{-3 / 4}(k) \log T}{T}\right) \\
=O\left(z^{-\beta} \sigma_{-3 / 4}(k) \log ^{2} T\right)+O\left(\frac{z^{2 \beta} \sigma_{-3 / 4}(k)}{T}\right)
\end{aligned}
$$

while

$$
\begin{aligned}
\Sigma_{B}= & O\left(\sum_{m=1}^{\infty} \frac{\mu^{2}(m)}{m^{\beta} \phi(m)}\right) \\
& +O\left(\frac{\log \log 10 M}{M^{\beta}} \sum_{M / 2 \leq m \leq 2 m} \frac{1}{|\log \{(M+1 / 2) / m\}|}\right) \\
= & O\left(\zeta(1+\beta) \sum_{m_{1}=1}^{\infty} \frac{\mu^{2}\left(m_{1}\right)}{m_{1}^{1+\beta} \phi\left(m_{1}\right)}\right)+O\left(\frac{\log \log 10 M \log 2 M}{M^{\beta}}\right) \\
= & O(1 / \beta)+O\left(\frac{\log ^{2} 2 z}{z^{\beta}}\right)=O(\log T)+O\left(\frac{\log ^{2} 2 z}{z^{\beta}}\right)
\end{aligned}
$$

by a familiar procedure in the theory of the Riemann zeta function (see Titchmarsh [10], p. 53 for an example). Let us then insert this with (50) and (51) in (49) to obtain

$$
M_{k}(s, z)=O\left\{\sigma_{-3 / 4}(k) \log ^{2} T\right\}+O\left(\frac{z^{2 \beta} \sigma_{-3 / 4}(k) \log T}{T}\right)+O\left(\frac{z^{\beta} \log ^{2} 2 z}{T}\right)
$$

whenever (42) and (47) hold, wherefore, now setting

$$
T=e^{A_{5} \sqrt{\log x}}
$$

for a sufficiently large positive constant $A_{5}$, we conclude that

$$
\begin{aligned}
M_{k}(s, z) & =O\left\{\sigma_{-3 / 4}(k) \log x\right\}+O\left\{\sigma_{-3 / 4}(k) e^{-A_{6} \sqrt{\log x}}\right\} \\
& =O\left\{\sigma_{-3 / 4}(k) \log x\right\}
\end{aligned}
$$

because of (36). 
The time has come to return to $\Gamma(s, R, \varrho)$ in $(43)$ and to deduce from (45) and (53) that it equals

$$
\begin{aligned}
O\left(\frac{\mu^{2}(\varrho) \varrho \log x}{\phi(\varrho)}\right. & \left.\sum_{\substack{\varrho^{\prime} \leq R / \varrho \\
\left(\varrho^{\prime}, \varrho\right)=1}} \frac{\mu^{2}\left(\varrho^{\prime}\right) \sigma_{-3 / 4}\left(\varrho \varrho^{\prime}\right)}{\phi\left(\varrho^{\prime}\right)}\right) \\
& =O\left(\frac{\mu^{2}(\varrho) \varrho \sigma_{-3 / 4}(\varrho) \log x}{\phi(\varrho)} \sum_{\varrho^{\prime} \leq R / \varrho} \frac{\mu^{2}\left(\varrho^{\prime}\right) \sigma_{-3 / 4}\left(\varrho^{\prime}\right)}{\phi\left(\varrho^{\prime}\right)}\right) \\
& =O\left\{\mu^{2}(\varrho) \sigma_{-3 / 4}^{2}(\varrho) \log ^{2} x\right\},
\end{aligned}
$$

which together with (43) and (44) shews that

$$
\begin{aligned}
V^{2}(R) F_{R}(s) & =O\left(\log ^{4} x \sum_{\varrho \leq R} \frac{\sigma_{-3 / 4}^{5}(\varrho)}{\varrho^{1-\beta}}\right)=O\left(\log ^{4} x \sum_{\varrho \leq R} \frac{\sigma_{-1 / 2}(\varrho)}{\varrho^{1-\beta}}\right) \\
& =O\left(\log ^{4} x \sum_{\varrho_{1} \leq R} \frac{1}{\varrho_{1}^{1-\beta}}\right)=O\left(\frac{R^{\beta} \log ^{4} x}{\beta}\right)
\end{aligned}
$$

when $s$ is on the contour of integration appertaining to $I_{x, R}^{(1)}$ in (39). Therefore

$$
\begin{aligned}
I_{x, R}^{(1)} & =O\left(\frac{R^{\beta} x^{2-\beta} \log ^{4} x \log T}{\beta} \int_{0}^{\infty} \frac{d t}{(1+t)^{2}}\right) \\
& =O\left(\frac{x^{2}(x / R)^{-\beta} \log ^{4} x \log T}{\beta}\right)=O\left(x^{2}(x / R)^{-\beta} \log ^{5} x\right)
\end{aligned}
$$

by (39), (36), and (38) so that

$$
I_{x, R}=O\left(x^{2}(x / R)^{-\beta} \log ^{5} x\right)+O\left(x^{2} e^{-A_{7} \sqrt{\log x}}\right)
$$

after estimating the last term in (41) as in the final derivation of (53).

The lemma is now available. Since certainly $(x / R)^{\beta}>\log ^{5} x$ when

$$
R<x\left(\log ^{5} x\right)^{-\left(A_{5} / A_{4}\right) \sqrt{\log x}}
$$

and hence when

$$
R<2 x \exp \left(-\log ^{3 / 5} x\right)
$$

we infer from (35) and (54) that

$$
\Sigma_{x, R}^{(1)}=\frac{1}{2} V(R) x^{2}+O(x)
$$

in the latter range of $R$. Hence, by a standard Tauberian argument involving the non-negativity of $\Lambda_{R}^{2}(n)$, we gain 
Lemma 2. In the notation of (13) and of Section 3 of XII, we have

$$
\sum_{n \leq x} \Lambda_{R}^{2}(n)=x V(R)+O\left(x \log ^{1 / 2} x\right)
$$

for

$$
1 \leq R \leq x \exp \left(-\log ^{3 / 5} x\right) .
$$

Thus the values of $\Sigma_{x, R}$ and $x V(R)$ are still closely associated for $R>$ $x \log ^{1 / 2} x$ despite the loss of formal connections between them that was mentioned in the preface to the proof. Their underlying identification is actually still to be foreseen once it is appreciated that our method can also shew that the quadratic forms in (27) and (29) are still almost equal even when $R$ is large, a feature that could form a partial basis of an alternative proof of the lemma provided we had recourse to a theorem of Axer's type. It is also worth observing that we can infer from the lemma that

$$
\pi(x)<\frac{(1+\varepsilon) x}{\log x} \quad\left(x>x_{0}(\varepsilon)\right),
$$

although we have of course used properties of $\zeta(s)$ that are tantamount to the prime number theorem (with the usual remainder term).

Finally, an easy estimate implicit in XII (16) is stated as

LEMma 3. We have

$$
\sum_{p \leq x} \log p \Lambda_{R}(p)=x \log R+O(x)+O(R \log R)
$$

for $R \leq x$.

4. The preliminary treatment. Both the unconditional and conditional treatments of the sum

$$
S(x, Q)=\sum_{k \leq Q} \sum_{\substack{0<a \leq k \\(a, k)=1}} E^{2}(x ; a, k)=\sum_{k \leq Q} G(x, k)
$$

in (2) for the initially chosen convenient range $x^{1 / 2} \log ^{9 / 2} x<Q \leq x$ have a common genesis involving two immediate simplifications of previous work brought about by the diminution of requirement from asymptotic formulae to lower bounds. First, since

$$
\sum_{\substack{0<a \leq k \\(a, k)=1}} \theta(x ; a, k)=\sum_{\substack{p \leq x \\ p \nmid k}} \log p=\theta_{k}(x), \quad \text { say, }
$$

and

$$
\theta_{k}(x)=\theta(x)-\sum_{\substack{p \leq x \\ p \mid k}} \log p=\theta(x)+O(\log k)
$$


we have

$$
\begin{aligned}
G(x, k) & \geq \sum_{\substack{0<a \leq k \\
(a, k)=1}} \theta^{2}(x ; a, k)-\frac{\theta_{k}^{2}(x)}{\phi(k)} \\
& =\sum_{0<a \leq k} \theta^{2}(x ; a, k)-\sum_{p \mid k} \log ^{2} p-\frac{\theta^{2}(x)}{\phi(k)}+O\left(\frac{x \log k}{\phi(k)}\right) \\
& =\sum_{0<a \leq k} \theta^{2}(x ; a, k)-\frac{\theta^{2}(x)}{\phi(k)}+O\left(\frac{x \log k}{\phi(k)}\right)
\end{aligned}
$$

for $x^{1 / 2}<k \leq x$ by the definition of $E(x ; a, k)$ implicit in (1) and by an elementary result in the theory of probability. Hence, revising the notation till the end of the proofs by writing $Q=Q_{2}$ and

$$
Q_{1}=Q_{2} / \log x
$$

so that

$$
x^{1 / 2} \log ^{9 / 2} x<Q_{2} \leq x \quad \text { and } \quad Q_{1}>x^{1 / 2} \log ^{7 / 2} x,
$$

we deduce that

$$
\begin{aligned}
S\left(x, Q_{2}\right) \geq & \sum_{Q_{1}<k \leq Q_{2}} G(x, k) \geq \sum_{Q_{1}<k \leq Q_{2}} \sum_{0<a \leq k} \theta^{2}(x ; a, k) \\
& -\theta^{2}(x) \sum_{Q_{1}<k \leq Q_{2}} \frac{1}{\phi(k)}+O\left(x \sum_{k \leq Q_{2}} \frac{\log k}{\phi(k)}\right) \\
= & \sum_{Q_{1}<k \leq Q_{2}} \sum_{0<a \leq k} \theta^{2}(x ; a, k)-\frac{\zeta(2) \zeta(3)}{\zeta(6)} \theta^{2}(x) \log \frac{Q_{2}}{Q_{1}} \\
& +O\left(\frac{x^{2} \log x}{Q_{1}}\right)+O\left(x \log ^{2} x\right) \\
= & T\left(x ; Q_{1}, Q_{2}\right)-\frac{\zeta(2) \zeta(3)}{\zeta(6)} \theta^{2}(x) \log \frac{Q_{2}}{Q_{1}}+O\left(Q_{2} x\right), \quad \text { say, }
\end{aligned}
$$

with the aid of the familiar asymptotic formula for

$$
\sum_{k \leq \xi} \frac{1}{\phi(k)}
$$

that is quoted, for example, in Lemma 1 of $\mathrm{I}$.

To bound $T\left(x ; Q_{1}, Q_{2}\right)$ from below, we bring in the partial substitute $\left(^{2}\right)$

$$
\Upsilon_{R}(x ; a, k)=\Upsilon_{R}(0, x ; a, k)=\sum_{\substack{n \leq x \\ n \equiv a, \bmod k}} \Lambda_{R}(n)
$$

$\left({ }^{2}\right)$ We use the notation $\Upsilon_{R}(x ; a, k)$ of Lemma 1 in preference to $\psi_{R}(x ; a, k)$ in XII to avoid confusion with the function $\psi(u)$ in (14). 
for $\theta(x ; a, k)$, where throughout $R$ will be governed in particular by the conditions

$$
R \leq x \exp \left(-\log ^{3 / 5} x\right), \quad R>x / Q_{1}
$$

apart from any others to be imposed later. Then

$$
\left\{\theta(x ; a, k)-\Upsilon_{R}(x ; a, k)\right\}^{2}=\theta^{2}(x ; a, k)-2 \theta(x ; a, k) \Upsilon_{R}(x ; a, k)+\Upsilon_{R}^{2}(x ; a, k)
$$

being non-negative as in Friedlander and Goldston [1] and XII, we have

$$
\begin{aligned}
T\left(x ; Q_{1}, Q_{2}\right) \geq & 2 \sum_{Q_{1}<k \leq Q_{2}} \sum_{0<a \leq k} \theta(x ; a, k) \Upsilon_{R}(x ; a, k) \\
& -\sum_{Q_{1}<k \leq Q_{2}} \sum_{0<a \leq k} \Upsilon_{R}^{2}(x ; a, k) \\
= & 2 T_{1}\left(x ; Q_{1}, Q_{2}\right)-T_{2}\left(x ; Q_{1}, Q_{2}\right), \quad \text { say, }
\end{aligned}
$$

the sums $T_{i}\left(x ; Q_{1}, Q_{2}\right)$ in which are initially treated by a variant of a routine used in earlier members of this series. First, by (59), the inner sum in $T_{1}\left(x ; Q_{1}, Q_{2}\right)$ equals

$$
\sum_{\substack{p-n \equiv 0, \bmod k \\ p, n \leq x}} \log p \Lambda_{R}(n)=\sum_{p \leq x} \log p \Lambda_{R}(p)+\sum_{\substack{p-n=L k \\ p, n \leq x}} \log p \Lambda_{R}(n)
$$

and therefore

$$
\begin{aligned}
& T_{1}\left(x ; Q_{1}, Q_{2}\right) \\
= & \left(Q_{2}-Q_{1}+O(1)\right) \sum_{p \leq x} \log p \Lambda_{R}(p)+\sum_{Q_{1}<k \leq Q_{2}} \sum_{\substack{p-n=L k \\
p, n \leq x}} \log p \Lambda_{R}(n) \\
= & Q_{2} x \log R+O\left(Q_{1} x \log R\right)+O\left(Q_{2} x\right)+\sum_{\substack { Q_{1}<k \leq Q_{2} \\
\begin{subarray}{c}{p-n=L k \\
p, n \leq x{ Q _ { 1 } < k \leq Q _ { 2 } \\
\begin{subarray} { c } { p - n = L k \\
p , n \leq x } }\end{subarray}} \log p \Lambda_{R}(n) \\
= & Q_{2} x \log R+O\left(Q_{2} x\right)+J_{1}\left(x ; Q_{1}, Q_{2}\right), \quad \text { say, }
\end{aligned}
$$

by Lemma 3, (60), and (56). Similarly, taking advantage of Lemma 2 and symmetry, we also find that

$$
\begin{aligned}
T_{2}\left(x ; Q_{1}, Q_{2}\right) \leq & Q_{2} x \log R+O\left(Q_{2} x \log ^{1 / 2} x\right) \\
& +2 \sum_{Q_{1}<k \leq Q_{2}} \sum_{\substack{n-m=l k>0 \\
n, m \leq x}} \Lambda_{R}(m) \Lambda_{R}(n) \\
= & Q_{2} x \log R+O\left(Q_{2} x \log ^{1 / 2} x\right)+2 J_{2}\left(x ; Q_{1}, Q_{2}\right), \quad \text { say, }
\end{aligned}
$$

which combines with (62), (61), and (58) to yield our initial conclusion in the form of the inequality 


$$
\begin{aligned}
S\left(x, Q_{2}\right) \geq & Q_{2} x \log R+2 J_{1}\left(x ; Q_{1}, Q_{2}\right)-2 J_{2}\left(x ; Q_{1}, Q_{2}\right) \\
& -\frac{\zeta(2) \zeta(3)}{\zeta(6)} \theta^{2}(x) \log \frac{Q_{2}}{Q_{1}}+O\left(Q_{2} x \log ^{1 / 2} x\right) .
\end{aligned}
$$

Finally, now temporarily redeploying when convenient the symbol $Q$ to denote either $Q_{1}$ or $Q_{2}$ with the result that

$$
x^{1 / 2} \log ^{7 / 2} x<Q \leq x \quad \text { and } \quad R>x / Q
$$

by (57) and (60), we set

$$
J_{i}(x, Q)=J_{i}(x ; Q, x) \quad(i=1,2)
$$

in order to write

$$
J_{i}\left(x ; Q_{1}, Q_{2}\right)=J_{i}\left(x, Q_{1}\right)-J_{i}\left(x, Q_{2}\right),
$$

after which manœuvre it is best to let the exposition bifurcate into separate treatments for the unconditional and conditional theorems.

5. The unconditional theorem. In deducing our first theorem from (64), we generally follow the pattern of the parent paper I both to ease the exposition and to highlight the effect of our reduced requirements on the sharpness of our estimations in unconditional circumstances. Accordingly, we shall still need the asymptotic formula

$$
\sum_{l<\xi}\left(1-\frac{l}{\xi}\right)^{2} \frac{1}{\phi(l)}=\frac{\zeta(2) \zeta(3)}{\zeta(6)} \log \xi+C_{1}+\frac{\log \xi}{\xi}+O(1 / \xi)
$$

that in slightly stronger form was stated in Lemma 1 of I, although the use of the prime number theorem for arithmetical progressions will be replaced by that of the prime number theorem itself in the strong forms

$$
\begin{aligned}
\theta(u) & =u+O\left\{u \exp \left(-\log ^{3 / 5-\varepsilon} u\right)\right\}, \\
\theta_{1}(u) & =\int_{0}^{u} \theta(t) d t=\frac{1}{2} u^{2}+O\left\{u^{2} \exp \left(-\log ^{3 / 5-\varepsilon} u\right)\right\} .
\end{aligned}
$$

First, (62) and (66) mean that $J_{1}(x, Q)$ is the sum of all terms $\log p \Lambda_{R}(n)$ answering to all quadruplets $k, l, p, n$ that satisfy either the conditions

$$
k>Q ; \quad p, n \leq x ; \quad n-p=l k ; \quad l>0
$$

or the similar conditions

$$
k>Q ; \quad p, n \leq x ; \quad p-n=l k ; \quad l>0,
$$

in both of which the inequality $l<x / Q$ is implied. Secondly, the contribution to $J_{1}(x, Q)$ related to $(71)$ equals

(73) $J_{1}^{*}(x, Q)=\sum_{l<x / Q} \sum_{p<x-l Q} \log p \sum_{\substack{p+l Q<n \leq x \\ n \equiv p, \bmod l}} \Lambda_{R}(n)=\sum_{l<x / Q} \Sigma_{C}, \quad$ say, 
while that springing from (72) is likewise equal to

$$
J_{1}^{\dagger}(x, Q)=\sum_{l<x / Q} \sum_{l Q<p \leq x} \log p \sum_{\substack{n<p-l Q \\ n \equiv p, \bmod l}} \Lambda_{R}(n)=\sum_{l<x / Q} \Sigma_{D}, \quad \text { say. }
$$

Since the innermost sum in the middle element of (73) is

$$
\frac{(x-l Q-p) v\{(l, p)\}}{\phi(l)}+O(R)
$$

by Lemma 1 and (65), we have

$$
\begin{aligned}
& \text { (76) } \quad \Sigma_{C}=\frac{1}{\phi(l)} \sum_{\substack{p<x-l Q \\
p \nmid l}}(x-l Q-p) \log p+O(R x) \\
& =\frac{1}{\phi(l)} \sum_{p<x-l Q}(x-l Q-p) \log p+O\left(\frac{x \log l}{\phi(l)}\right)+O(R x) \\
& =\frac{\theta_{1}(x-l Q)}{\phi(l)}+O(R x)=\frac{(x-l Q)^{2}}{2 \phi(l)}+O\left\{x^{2} \exp \left(-\log ^{3 / 5-\varepsilon} x\right)\right\}
\end{aligned}
$$

in view of (60) and (70); likewise, but slightly less easily, we also have from (74) that

$$
\begin{aligned}
\Sigma_{D}= & \frac{1}{\phi(l)} \sum_{l Q<p \leq x}(p-l Q) \log p+O\left(\frac{x \log l}{\phi(l)}\right)+O(R x) \\
= & \frac{1}{\phi(l)}\left((x-l Q) \sum_{p \leq x} \log p-\sum_{p \leq x}(x-p) \log p\right. \\
& \left.+\sum_{p \leq l Q}(l Q-p) \log p\right)+O(R x) \\
= & \frac{1}{\phi(l)}\left\{(x-l Q) \theta(x)-\theta_{1}(x)+\theta_{1}(l Q)\right\}+O(R x) \\
= & \frac{1}{\phi(l)}\left\{(x-l Q) x-\frac{1}{2} x^{2}+\frac{1}{2} l^{2} Q^{2}\right\}+O\left\{x^{2} \exp \left(-\log ^{3 / 5-\varepsilon} x\right)\right\} \\
= & \frac{(x-l Q)^{2}}{2 \phi(l)}+O\left\{x^{2} \exp \left(-\log ^{3 / 5-\varepsilon} x\right)\right\} .
\end{aligned}
$$

Hence, joining (73) and (74) together after the above estimates have been embodied in them, we conclude that

$$
J_{1}(x, Q)=\sum_{l<x / Q} \frac{(x-l Q)^{2}}{\phi(l)}+O\left(\frac{x^{3}}{Q} \exp \left(-\log ^{3 / 5-\varepsilon} x\right)\right) .
$$

The estimation of $J_{2}(x, Q)$ is similar to that of $J_{1}(x, Q)$ save that there is only one constituent to be treated. Being equal to the sum of all terms $\Lambda_{k}(m) \Lambda_{R}(n)$ corresponding to the solutions of the conditions derived from 
(71) by substituting $m$ for $p$, the sum $J_{2}(x, Q)$ is shewn by Lemma 1 to equal

$$
\begin{aligned}
& \sum_{l<x / Q} \sum_{m<x-l Q} \Lambda_{R}(m) \sum_{\substack{m+l Q<n \leq x \\
n \equiv m, \bmod l}} \Lambda_{R}(n) \\
= & \sum_{l<x / Q} \frac{1}{\phi(l)} \sum_{\substack{m<x-l Q \\
(m, l)=1}}(x-l Q-m) \Lambda_{R}(m)+O\left(R \sum_{l<x / Q} \sum_{m \leq x}\left|\Lambda_{R}(m)\right|\right) \\
= & \sum_{l<x / Q} \frac{1}{\phi(l)} \sum_{\substack{m<x-l Q \\
(m, l)=1}}(x-l Q-m) \Lambda_{R}(m)+O\left(\frac{R x^{2} \log ^{2} x}{Q}\right)
\end{aligned}
$$

because

$$
\Lambda_{R}(m)=O\{d(m) \log x\}
$$

by (13) and (14) in XII. Next Lemma 1 also demonstrates that both

$$
\sum_{m \leq u} \Lambda_{R}(m)=u+O(R)
$$

and that

$$
\sum_{\substack{m \leq u \\ m \equiv 0, \bmod \delta}} \Lambda_{R}(m)=O(R)
$$

when $1<\delta \leq R$ and, in particular, when $\delta$ is a divisor of $l$ exceeding 1 . Hence the inner sum on the final line of (79) is

$$
\frac{1}{2}(x-l Q)^{2}+O\{R x d(l)\}
$$

by a simple combinatorial argument followed by integration, wherefore (79) produces

$$
\begin{aligned}
J_{2}(x, Q) & =\frac{1}{2} \sum_{l<x / Q} \frac{(x-l Q)^{2}}{\phi(l)}+O\left(\frac{R x^{2} \log ^{2} x}{Q}\right)+O\left(R x \sum_{l<x / Q} \frac{d(l)}{\phi(l)}\right) \\
& =\frac{1}{2} \sum_{l<x / Q} \frac{(x-l Q)^{2}}{\phi(l)}+O\left(\frac{R x^{2} \log ^{2} x}{Q}\right)+O\left(R x \log ^{2} x\right)
\end{aligned}
$$

and hence via (60) the estimate

$$
J_{2}(x, Q)=\frac{1}{2} \sum_{l<x / Q} \frac{(x-l Q)^{2}}{\phi(l)}+O\left(\frac{x^{3}}{Q} \exp \left(-\log ^{3 / 5-\varepsilon} x\right)\right)
$$

that is parallel to (78). 
The proof is almost complete. All we have to do is first to deploy (78), (82), and (68) in the evaluation of $2 J_{1}(x, Q)-2 J_{2}(x, Q)$ as

$$
\begin{aligned}
\sum_{l<x / Q} \frac{(x-l Q)^{2}}{\phi(l)}+O & \left(\frac{x^{3}}{Q} \exp \left(-\log ^{3 / 5-\varepsilon} x\right)\right) \\
= & \frac{\zeta(2) \zeta(3)}{\zeta(6)} x^{2} \log \frac{x}{Q}+C_{1} x^{2}+Q x \log \frac{x}{Q}+O(x Q) \\
& +O\left(\frac{x^{3}}{Q} \exp \left(-\log ^{3 / 5-\varepsilon} x\right)\right)
\end{aligned}
$$

and then to point this at $(67)$ to shew that the quantity $2 J_{1}\left(x ; Q_{1}, Q_{2}\right)-$ $2 J_{2}\left(x ; Q_{1}, Q_{2}\right)$ in (64) equals

$$
\begin{aligned}
\frac{\zeta(2) \zeta(3)}{\zeta(6)} x^{2} \log \frac{Q_{2}}{Q_{1}}-Q_{2} x \log \frac{x}{Q_{2}}+O\left(Q_{1} x \log x\right) & +O\left(Q_{2} x\right) \\
& +O\left(\frac{x^{3}}{Q_{1}} \exp \left(-\log ^{3 / 5-\varepsilon} x\right)\right) \\
=\frac{\zeta(2) \zeta(3)}{\zeta(6)} x^{2} \log \frac{Q_{2}}{Q_{1}}-Q_{2} x \log \frac{x}{Q_{2}}+O\left(Q_{2} x\right) & +O\left(\frac{x^{3}}{Q_{2}} \exp \left(-\log ^{3 / 5-\varepsilon} x\right)\right)
\end{aligned}
$$

in virtue of (56) and our conventions regarding the use of the $\varepsilon$ symbol. Thus, by the prime number theorem in (69), we infer that

$$
\begin{aligned}
S\left(x, Q_{2}\right)>Q_{2} x \log \frac{R Q_{2}}{x}+O\left(Q_{2} x \log ^{1 / 2} x\right) & +O\left(\frac{x^{3}}{Q_{2}} \exp \left(-\log ^{3 / 5-\varepsilon} x\right)\right) \\
& +O\left(x^{2} \exp \left(-\log ^{3 / 5-\varepsilon} x\right)\right),
\end{aligned}
$$

which for $Q_{2}>x \exp \left(-\log ^{3 / 5-\varepsilon_{1}} x\right)$ implies that

$$
\begin{aligned}
S\left(x, Q_{2}\right) & >Q_{2} x \log \frac{R Q_{2}}{x}+O\left(Q_{2} x \log ^{1 / 2} x\right) \\
& >\left(1-\varepsilon_{2}\right) Q_{2} x \log x>\left(1-\varepsilon_{2}\right) Q_{2} x \log Q_{2} \quad\left(x>x_{0}\left(\varepsilon_{1}, \varepsilon_{2}\right)\right)
\end{aligned}
$$

on our setting $R=x \exp \left(-\log ^{3 / 5} x\right)$ in conformity with (55). Thus, reverting to the original meaning of $Q$, we have established

THEOREM 1. Let $E(x ; a, k)$ be defined as in (1) above and suppose that $\varepsilon_{1}, \varepsilon_{2}$ are any (arbitrarily small) positive constants. Then, for

$$
x \exp \left\{-\log ^{3 / 5-\varepsilon_{1}} x\right\}<Q \leq x \quad \text { and } \quad x>x_{0}\left(\varepsilon_{1}, \varepsilon_{2}\right),
$$

we have

$$
\sum_{k \leq Q} \sum_{\substack{0<a \leq k \\(a, k)=1}} E^{2}(x ; a, k)>\left(1-\varepsilon_{2}\right) Q x \log Q .
$$


6. The conditional theorem-first part of the treatment. We now consider the impact on our work of assuming a weak version of the Riemann hypothesis to the effect that $\zeta(s)$ has no zeros $\varrho=\beta+i \gamma$ for which $\beta>3 / 4$, which supposition implies that

$$
\theta(x)=x+O\left(x^{3 / 4} \log ^{2} x\right)
$$

by the classical theory. To take maximum advantage of the new circumstances it is necessary to reconsider the effect on our calculations of the entry into our work of both the explicit (first) term and the remainder (second) term in Lemma 1. So far as the main term is concerned, we need only reappraise at (73), (74), (76), and (77) the previous treatment, which for comparative reasons and lucidity had been modelled on that of I. On the other hand, all of (76), (77), and (79) are involved when we usually employ a large sieve method to assess the implication of replacing the remainder term in Lemma 1 by its alternative formulation in terms of the functions $\psi(u)$ and $\psi^{-}(u)$.

The first category of revision arises at the second line of (76) and the first line of (77), in each of which the first two terms flow from the explicit term in Lemma 1. Since the second items $O\{x \log l / \phi(l)\}$ produce a satisfactory contribution

$$
O\left(x \sum_{l<x / Q} \frac{\log l}{\phi(l)}\right)=O\left(x \log ^{2} x\right)
$$

after summation over $l$, we are left with the sum over $l<x / Q$ of

$$
\frac{1}{\phi(l)}\left(\sum_{p<x-l Q}(x-l Q-p) \log p+\sum_{l Q<p \leq x}(p-l Q) \log p\right),
$$

for which the previous method of development becomes inadequate unless it be boosted by the use of the explicit formulae for $\theta(x)$ and $\theta_{1}(x)$ in terms of the zeros $\varrho$. But such a procedure involves an unnecessary excursion and is best avoided by changing the order of summations in $p$ and $l$ so as to obtain the quantity

$$
\sum_{p<x-Q} \log p \sum_{l<(x-p) / Q} \frac{x-p-l Q}{\phi(l)}+\sum_{Q<p \leq x} \log p \sum_{l<p / Q} \frac{p-l Q}{\phi(l)},
$$

wherein the inner sums are evaluated by the formula

$$
\sum_{l<\xi}\left(1-\frac{l}{\xi}\right) \frac{1}{\phi(l)}=\frac{\zeta(2) \zeta(3)}{\zeta(6)} \log \xi+C_{2}+\frac{\log \xi}{2 \xi}+O(1 / \xi)
$$


that is similar to (68). Having thereby identified this contribution as

$$
\begin{aligned}
& \frac{\zeta(2) \zeta(3)}{\zeta(6)}\left(\sum_{p<x-Q}(x-p) \log p \log \frac{C_{3}(x-p)}{Q}+\sum_{Q<p \leq x} p \log p \log \frac{C_{3} p}{Q}\right) \\
& +\frac{1}{2} Q\left(\sum_{p<x-Q} \log p \log \frac{x-p}{Q}+\sum_{Q<p \leq x} \log p \log \frac{p}{Q}\right)+O\left(Q \sum_{p \leq x} \log p\right) \\
& =\frac{\zeta(2) \zeta(3)}{\zeta(6)}\left(\sum_{p<x-Q}(x-p) \log p \log \frac{C_{3}(x-p)}{Q}+\sum_{p \leq x} p \log p \log \frac{C_{3} p}{Q}\right) \\
& +\frac{1}{2} Q\left(\sum_{p<x-Q} \log p \log \frac{x-p}{Q}+\sum_{p \leq x} \log p \log \frac{p}{Q}\right) \\
& +O\left(Q \sum_{p \leq Q} \log p \log \frac{A_{8} Q}{p}\right)+O(Q x),
\end{aligned}
$$

we use the prime number theorem (in a simple unconditional form) with partial summation (or parallel procedure) to estimate the antepenultimate and penultimate parentheses as

$$
x \log \frac{x}{Q}+x \log \frac{x}{Q}+O(x)=2 x \log \frac{x}{Q}+O(x)
$$

and

$$
O\left(Q^{2}\right)
$$

respectively, and consequently obtain

$$
\begin{array}{r}
\frac{\zeta(2) \zeta(3)}{\zeta(6)}\left(\sum_{p<x-Q}(x-p) \log p \log \frac{C_{3}(x-p)}{Q}+\right. \\
\left.\sum_{p \leq x} p \log p \log \frac{C_{3} p}{Q}\right) \\
+Q x \log \frac{x}{Q}+O(Q x),
\end{array}
$$

the influence of which on $J_{1}\left(x ; Q_{1}, Q_{2}\right)$ is

$$
\begin{aligned}
\frac{\zeta(2) \zeta(3)}{\zeta(6)} & \left(\sum_{p<x-Q_{1}}(x-p) \log p \log \frac{C_{3}(x-p)}{Q_{1}}\right. \\
& \left.-\sum_{p<x-Q_{2}}(x-p) \log p \log \frac{C_{3}(x-p)}{Q_{2}}+\log \frac{Q_{2}}{Q_{1}} \sum_{p \leq x} p \log p\right) \\
& -Q_{2} x \log \frac{x}{Q_{2}}+O\left(Q_{2} x\right)
\end{aligned}
$$

by (67) and (56). But the difference between the first two sums in this is 


$$
\begin{gathered}
\log \frac{Q_{2}}{Q_{1}} \sum_{p<x-Q_{2}}(x-p) \log p+\sum_{x-Q_{2} \leq p<x-Q_{1}}(x-p) \log p \log \frac{C_{3}(x-p)}{Q_{1}} \\
=\log \frac{Q_{2}}{Q_{1}} \sum_{p \leq x}(x-p) \log p+O\left(x \log x \log \frac{Q_{2}}{Q_{1}} \sum_{x-Q_{2}<p \leq x} 1\right) \\
=\log \frac{Q_{2}}{Q_{1}} \sum_{p \leq x}(x-p) \log p+O\left(Q_{2} x \log \frac{Q_{2}}{Q_{1}} \cdot \frac{\log x}{\log Q_{2}}\right) \\
=\log \frac{Q_{2}}{Q_{1}} \sum_{p \leq x}(x-p) \log p+O\left(Q_{2} x \log \frac{Q_{2}}{Q_{1}}\right)
\end{gathered}
$$

by (56), (57), and an upper bound sieve method. Placed in (85), this together with (84) produces

$$
\begin{aligned}
\text { (86) } \quad & \frac{\zeta(2) \zeta(3)}{\zeta(6)} x \log \frac{Q_{2}}{Q_{1}} \sum_{p \leq x} \log p-Q_{2} x \log \frac{x}{Q_{2}}+O\left(Q_{2} x \log \frac{Q_{2}}{Q_{1}}\right) \\
+ & O\left(x \log ^{2} x\right)=\frac{\zeta(2) \zeta(3)}{\zeta(6)} x \theta(x) \log \frac{Q_{2}}{Q_{1}}-Q_{2} x \log \frac{x}{Q_{2}}+O\left(Q_{2} x \log \frac{Q_{2}}{Q_{1}}\right)
\end{aligned}
$$

as the effect of the main term in Lemma 1 on our calculation of $J_{1}\left(x ; Q_{1}, Q_{2}\right)$ when (56) and (57) are still assumed.

In revising our assessment of the influence of Lemma 1 on our treatment of $J_{2}\left(x ; Q_{1}, Q_{2}\right)$, we only ignore at this stage its remainder term at its first appearance in (79) because it merely creates at its second coming a contribution $O\left(R x \log ^{2} x\right)$ in (81) of acceptable size. Thus the appropriate part of $J_{2}\left(x ; Q_{1}, Q_{2}\right)$ attained is the combination

$$
\begin{aligned}
\frac{\zeta(2) \zeta(3)}{2 \zeta(6)} x^{2} \log \frac{Q_{2}}{Q_{1}}-\frac{1}{2} Q_{2} x \log & \frac{x}{Q_{2}}+O\left(Q_{2} x\right) \\
& +O\left(Q_{1} x \log \frac{x}{Q_{1}}\right)+O\left(R x \log ^{2} x\right) \\
= & \frac{\zeta(2) \zeta(3)}{2 \zeta(6)} x^{2} \log \frac{Q_{2}}{Q_{1}}-\frac{1}{2} Q_{2} x \log \frac{x}{Q_{2}}+O\left(Q_{2} x\right)+\left(R x \log ^{2} x\right)
\end{aligned}
$$

of the differences of the relevant terms on the right of (81) after (67) and (68) have been applied.

Altogether, therefore, the culmination of substantially ignoring the remainder term in Lemma 1 when estimating $S\left(x, Q_{2}\right)$ is the construction of the portion

$$
\begin{aligned}
Q_{2} x \log R-\frac{\zeta(2) \zeta(3)}{\zeta(6)}\left(x^{2}-2 x \theta(x)\right. & \left.+\theta^{2}(x)\right) \log \frac{Q_{2}}{Q_{1}}-Q_{2} x \log \frac{x}{Q_{2}} \\
& +O\left(Q_{2} x \log ^{1 / 2} x\right)+O\left(R x \log ^{2} x\right)
\end{aligned}
$$




$$
\begin{aligned}
= & Q_{2} x \log \frac{R Q_{2}}{x}-\frac{\zeta(2) \zeta(3)}{\zeta(6)}\{x-\theta(x)\}^{2} \log \frac{Q_{2}}{Q_{1}} \\
& +O\left(Q_{2} x \log ^{1 / 2} x\right)+O\left(R x \log ^{2} x\right) \\
= & Q_{2} x \log \frac{R Q_{2}}{x}+O\left(x^{3 / 2} \log ^{5} x\right)+O\left(Q_{2} x \log ^{1 / 2} x\right)+O\left(R x \log ^{2} x\right) \\
= & Q_{2} x \log \frac{R Q_{2}}{x}+O\left(Q_{2} x \log ^{1 / 2} x\right)+O\left(R x \log ^{2} x\right),
\end{aligned}
$$

whether we arrive via (64), (86), (87), (83), and (57).

7. The application of the large sieve. If we shall benefit from the improvements gained in the previous section through the weak form of the Riemann hypothesis, we must now use the second form of Lemma 1 in order to obtain better estimates in that part of the analysis that does not appertain to the main term in that lemma. This aspect of the work is, however, completely unconditional and depends mainly on a Fourier analysis of the functions $\psi(u), \psi^{-}(u)$ and on a large sieve method, the main results on which are given in the following lemmata.

LEMMA 4. Let $v_{1}, \ldots, v_{r}, \ldots, v_{s}$ be a set of real numbers, each of which is to be affected by a real weight $w_{r}$. Then, for any positive integer $N \geq 2$,

$$
\begin{aligned}
\sum_{1 \leq r \leq s} w_{r} \psi\left(v_{r}\right)= & O\left(\frac{1}{N} \sum_{1 \leq r \leq s}\left|w_{r}\right|\right) \\
& +O\left\{\sum_{1 \leq h \leq N} \frac{1}{h}\left(\left|\sum_{1 \leq r \leq s} w_{r} e^{2 \pi i h v_{r}}\right|+\left|\sum_{1 \leq r \leq s}\right| w_{r}\left|e^{2 \pi i h v_{r}}\right|\right)\right\} .
\end{aligned}
$$

The function $\psi\left(v_{r}\right)$ in this formula may be replaced by $\psi^{-}\left(v_{r}\right)$.

This supplies one of the more accurate estimates of its type, although older results in the same genre would serve us equally well here. It depends on Vaaler's approximation to $\psi(u)$ given in his Theorem A.6 of [11] and its restatement as

$$
\psi(u)=\sum_{0<|h| \leq N} c_{h} e^{2 \pi i h u}+O\left(\frac{1}{N}+\sum_{0<h \leq N} c_{h}^{\prime} e^{2 \pi i h u}\right),
$$

wherein

$$
c_{h}, c_{h}^{\prime}=O(1 /|h|)
$$

and the trigonometrical series within the $O$-symbol is non-negative. This shews that the left hand side of the proposed formula equals

$$
\sum_{1 \leq r \leq s} w_{r} \sum_{0<|h| \leq N} c_{h} e^{2 \pi i h v_{r}}+O\left\{\sum_{1 \leq r \leq s}\left|w_{r}\right|\left(\frac{1}{N}+\sum_{0<|h| \leq N} c_{h}^{\prime} e^{2 \pi i h v_{r}}\right)\right\}
$$




$$
\begin{aligned}
= & \sum_{0<|h| \leq N} c_{h} \sum_{1 \leq r \leq s} w_{r} e^{2 \pi i h v_{r}} \\
& +O\left\{\frac{1}{N} \sum_{1 \leq r \leq s}\left|w_{r}\right|+\sum_{0<|h| \leq N} c_{h}^{\prime} \sum_{1 \leq r \leq s}\left|w_{r}\right| e^{2 \pi i h v_{r}}\right\} \\
= & \left(\frac{1}{N} \sum_{1 \leq r \leq s}\left|w_{r}\right|\right) \\
& +O\left\{\sum_{0<h \leq N} \frac{1}{h}\left(\left|\sum_{1 \leq r \leq s} w_{r} e^{2 \pi i h v_{r}}\right|+\left|\sum_{1 \leq r \leq s}\right| w_{r}\left|e^{2 \pi i h v_{r}}\right|\right)\right\},
\end{aligned}
$$

as required.

Lemma 5. If $W(u, \theta)$ be defined as either

$$
\sum_{m \leq u} a_{m} e^{2 \pi i m \theta} \text { or } \sum_{m<u} a_{m} e^{2 \pi i m \theta}
$$

for a sequence of real numbers $a_{1}, \ldots, a_{m}, \ldots$, then

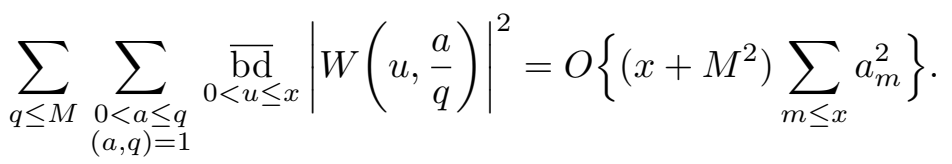

We have stated this best possible version of the maximal large sieve inequality in the form discovered and proved by Montgomery [9], even though earlier estimates containing a superfluous logarithm would suffice in the present context.

We first consider the sum $J_{1}^{*}(x, Q)$ in $(73)$ and appraise the consequence of replacing for $p \nmid l$ the remainder term $O(R)$ in $(75)$ by the term

$$
\sum_{\substack{d \leq R \\(d, l)=1}} V(R) \lambda_{d}\left\{\psi\left(\frac{x}{l d}-\frac{p \bar{d}}{l}\right)-\psi\left(\frac{Q}{d}+\frac{p}{l d}-\frac{p \bar{d}}{l}\right)\right\}
$$

given in the second form of Lemma 1 , noting that in the opposite case $p \mid l$ we may simply add the latter term to the right of (75) without disturbing its validity. The contribution of the entities $O(R)$ remaining being

$$
O\left(R \sum_{l<x / Q} \sum_{\substack{p \leq x \\ p \mid l}} \log p\right)=O\left(R \sum_{l<x / Q} \log l\right)=O\left(\frac{R x \log x}{Q}\right),
$$

we then prepare for the summation of (89) over $p$ and $l$ by observing that

$$
\psi\left(\frac{Q}{d}+\frac{p}{l d}-\frac{p \bar{d}}{l}\right)=\psi\left(\frac{Q}{d}+\frac{p \bar{l}}{d}\right)
$$


because of the identical congruence $d \bar{d}+l \bar{l} \equiv 1, \bmod d l$, that involves solutions $\bar{d}$ and $\bar{l}$ of the respective congruences $d \bar{d} \equiv 1, \bmod l$, and $l \bar{l} \equiv 1, \bmod d$. The donation to $J_{1}^{*}(x, Q)$ to be considered is therefore

$$
\begin{aligned}
\sum_{l<x / Q} \sum_{\substack{d \leq R \\
(d, l)=1}} V(R) \lambda_{d} & \sum_{p<x-l Q} \log p \psi\left(\frac{x}{l d}-\frac{p \bar{d}}{l}\right) \\
& -\sum_{l<x / Q} \sum_{\substack{d \leq R \\
(d, l)=1}} V(R) \lambda_{d} \sum_{p<x-l Q} \log p \psi\left(\frac{Q}{d}+\frac{p \bar{l}}{d}\right),
\end{aligned}
$$

which by Lemma 4 and XII (13) is equal to

$$
\begin{aligned}
& O\left(\frac{1}{N} \sum_{l<x / Q} \sum_{d \leq R} \log \frac{2 R}{d} \sum_{p \leq x} \log p\right) \\
& +O\left(\log x \sum_{0<h \leq N} \frac{1}{h} \sum_{l<x / Q} \sum_{\substack{d \leq R \\
(d, l)=1}}\left|\sum_{p<x-l Q} \log p e^{2 \pi i h \bar{d} p / l}\right|\right) \\
& +O\left(\log x \sum_{0<h \leq N} \frac{1}{h} \sum_{l<x / Q} \sum_{\substack{d \leq R \\
(d, l)=1}}\left|\sum_{p<x-l Q} \log p e^{2 \pi i h \bar{l} p / d}\right|\right) \\
& =O\left(\frac{x^{2} R}{Q N}\right)+O\left(\log x \sum_{0<h \leq N} \frac{1}{h} \Sigma_{h, 1}\right)+O\left(\log x \sum_{0<h \leq N} \frac{1}{h} \Sigma_{h, 2}\right) \\
& =O(1)+O\left\{\log x J_{1}^{(1)}(x, Q)\right\}+O\left\{\log x J_{1}^{(2)}(x, Q)\right\}, \quad \text { say, }
\end{aligned}
$$

when

$$
N=[x]^{3} .
$$

To handle the sums $J_{1}^{(i)}(x, Q)$ let us specialize the subject $W(u, \theta)$ of Lemma 5 as

$$
\sum_{p<u} \log p e^{2 \pi i p \theta}
$$

and then write

$$
\begin{aligned}
\Sigma_{h, 1} & =\sum_{l<x / Q} \sum_{\substack{d \leq R \\
(d, l)=1}}\left|W\left(x-l Q, \frac{h \bar{d}}{l}\right)\right| \\
& =\sum_{\substack{\delta \mid h \\
\delta<x / Q}} \sum_{\substack{l<x / Q \\
(l, h)=\delta}} \sum_{\substack{d \leq R \\
(d, l)=1}}\left|W\left(x-l Q, \frac{h \bar{d}}{l}\right)\right|
\end{aligned}
$$




$$
\begin{aligned}
& \leq \sum_{\substack{\delta \mid h \\
\delta<x / Q}} \sum_{\substack{l_{1}<x /(Q \delta) \\
\left(l_{1}, h / \delta\right)=1}} \sum_{\substack{d \leq R \\
\left(d, l_{1}\right)=1}}\left|W\left(x-l_{1} \delta Q, \frac{(h / \delta) \bar{d}}{l_{1}}\right)\right| \\
& =\sum_{\substack{\delta \mid h \\
\delta<x / Q}} \Sigma_{h, 1, \delta}, \quad \text { say, }
\end{aligned}
$$

where $\bar{d}$ may now merely be defined, $\bmod l_{1}$, by $d \bar{d} \equiv 1, \bmod l_{1}$. Next, since the second part of (65) is yet assumed and since therefore the number $(h / \delta) \bar{d}$ related to the innermost sum ranges through at most $2 R / l_{1}$ complete sets of reduced residues, $\bmod l_{1}$,

$$
\Sigma_{h, 1, \delta} \leq 2 R \sum_{l_{1}<x /(Q \delta)} \frac{1}{l_{1}} \sum_{\substack{0<a \leq l_{1} \\\left(a, l_{1}\right)=1}}\left|W\left(x-l_{1} \delta Q, \frac{a}{l_{1}}\right)\right|,
$$

wherefore, by the Cauchy-Schwarz inequality and Lemma 5 ,

$$
\text { (94) } \begin{aligned}
\Sigma_{h, 1, \delta} \leq & 2 R\left(\sum_{l_{1}<x /(Q \delta)} \frac{1}{l_{1}^{2}} \sum_{\left.\substack{0<a \leq l_{1} \\
l_{1}<x /(Q \delta)}\right)^{1 / 2}} \sum_{\substack{0<a \leq l_{1} \\
\left(a, l_{1}\right)=1}}\left|W\left(x-l_{1} \delta Q, \frac{a}{l_{1}}\right)\right|^{2}\right)^{1 / 2} \\
= & O\left\{R\left(\sum_{l_{1}<x /(Q \delta)} \frac{1}{l_{1}}\right)^{1 / 2}\left(\frac{x^{2}}{Q^{2} \delta^{2}}+x\right)^{1 / 2}\left(\sum_{p \leq x} \log ^{2} p\right)^{1 / 2}\right\} \\
= & O(R x \log x)
\end{aligned}
$$

because of the first part of (65). From this, (93), (91), and (92) we first infer that

$$
\Sigma_{h, 1}=O(R x d(h) \log x)
$$

and then that

$$
J_{1}^{(1)}(x, Q)=O\left(R x \log x \sum_{0<h \leq N} \frac{d(h)}{h}\right)=O\left(R x \log ^{3} x\right),
$$

thus completing the estimation of the second constituent in (91).

The analysis of $J_{1}^{(2)}(x, Q)$ resembles that of $J_{1}^{(1)}(x, Q)$, although our interchanging the rôles of $l$ and $d$ slightly lengthens the calculations. Now, by (91) and an alteration in the order of summations, 


$$
\begin{aligned}
\Sigma_{h, 2} & =\sum_{d \leq R} \sum_{\substack{l<x / Q \\
(l, d)=1}}\left|W\left(x-l Q, \frac{h \bar{l}}{d}\right)\right| \\
& =\sum_{\substack{\delta \mid h \\
\delta \leq R}} \sum_{\substack{d \leq R \\
(d, h)=\delta}} \sum_{\substack{l<x / Q \\
(l, d)=1}}\left|W\left(x-l Q, \frac{h \bar{l}}{d}\right)\right| \\
& \leq \sum_{\substack{\delta \mid h \\
\delta \leq R}} \sum_{\substack{d_{1} \leq R / \delta \\
\left(d_{1}, h / \delta\right)=1}} \sum_{\substack{l<x / Q \\
\left(l, d_{1}\right)=1}}\left|W\left(x-l Q, \frac{(h / \delta) \bar{l}}{d_{1}}\right)\right| \\
& =\sum_{\substack{\delta \mid h \\
\delta \leq R}} \sum_{h, 2, \delta}, \text { say, }
\end{aligned}
$$

the treatment of whose inner sum depends on whether $R / \delta \leq x / Q$ or $R / \delta>$ $x / Q$. In the former situation the estimate

$$
\begin{aligned}
\Sigma_{h, 2, \delta} & \leq \frac{2 x}{Q} \sum_{d_{1} \leq R / \delta} \frac{1}{d_{1}} \sum_{\substack{0<a \leq d_{1} \\
\left(a, d_{1}\right)=1}} \underset{\substack{\mathrm{bd} \\
0<u \leq x}}{ }\left|W\left(u, \frac{a}{d_{1}}\right)\right| \\
& =O\left\{\frac{x}{Q}\left(\sum_{d_{1} \leq R / \delta} \frac{1}{d_{1}}\right)^{1 / 2}\left(\frac{R^{2}}{\delta^{2}}+x\right)^{1 / 2}\left(\sum_{p \leq x} \log ^{2} p\right)^{1 / 2}\right\} \\
& =O\left(\frac{x^{2} \log x}{Q}\right)
\end{aligned}
$$

is obtained as in the derivation of (94). But in the latter situation we split $\Sigma_{h, 2, \delta}$ by the decomposition

$$
\Sigma_{h, 2, \delta}=\sum_{\begin{array}{c}
d_{1} \leq x / Q \\
\left(d_{1}, h / \delta\right)=1
\end{array}}+\sum_{\substack{x / Q<d_{1} \leq R / \delta \\
\left(d_{1}, h / \delta\right)=1}}=\Sigma_{h, 2, \delta, 1}+\Sigma_{h, 2, \delta, 2}, \quad \text { say, }
$$

wherein the assessment

$$
\Sigma_{h, 2, \delta, 1}=O\left(\frac{x^{2} \log x}{Q}\right)
$$

is a particular example of $(98)$ with $R / \delta$ replaced by $x / Q$. As for the other constituent in (99), on using the Cauchy-Schwarz inequality in a different way from before, we obtain

$$
\Sigma_{h, 2, \delta, 2} \leq\left(\sum_{\substack{d_{1} \leq R / \delta \\ l<x / Q}} 1\right)^{1 / 2}\left(\sum_{x / Q<d_{1} \leq R / \delta} \sum_{\substack{0<a \leq d_{1} \\\left(a, d_{1}\right)=1}} \overline{\substack{\mathrm{bd} \\ 0<u \leq x}}\left|W\left(u, \frac{a}{d}\right)\right|^{2}\right)^{1 / 2}
$$




$$
\begin{aligned}
& =O\left\{\left(\frac{R x}{Q \delta}\right)^{1 / 2}\left(\frac{R^{2}}{\delta^{2}}+x\right)^{1 / 2}\left(\sum_{p \leq x} \log ^{2} p\right)^{1 / 2}\right\} \\
& =O\left(\frac{R^{3 / 2} x \log x}{Q^{1 / 2} \delta^{3 / 2}}\right)+O\left(\frac{R^{1 / 2} x^{3 / 2} \log x}{Q^{1 / 2} \delta^{1 / 2}}\right) .
\end{aligned}
$$

The combination of this fact with (97) and (100), (99), (98), (65) first yields

$$
\begin{aligned}
\Sigma_{h, 2}= & O\left(\frac{d(h) x^{2} \log x}{Q}\right)+O\left(\frac{d(h) R^{3 / 2} x \log x}{Q^{1 / 2}}\right) \\
& +O\left(\frac{d(h) R^{1 / 2} x^{3 / 2} \log x}{Q^{1 / 2}}\right) \\
= & O\left(\frac{d(h) R^{3 / 2} x \log x}{Q^{1 / 2}}\right)+O\left(\frac{d(h) R^{1 / 2} x^{3 / 2} \log x}{Q^{1 / 2}}\right)
\end{aligned}
$$

and then $\left({ }^{3}\right)$, via $(91)$,

$$
\begin{aligned}
J_{1}^{(2)}(x, Q)= & O\left(\frac{R^{3 / 2} x \log x}{Q^{1 / 2}} \sum_{1 \leq h \leq N} \frac{d(h)}{h}\right) \\
& +O\left(\frac{R^{1 / 2} x^{3 / 2} \log x}{Q^{1 / 2}} \sum_{1 \leq h \leq N} \frac{d(h)}{h}\right) \\
= & O\left(\frac{R^{3 / 2} x \log ^{3} x}{Q^{1 / 2}}\right)+O\left(\frac{R^{1 / 2} x^{3 / 2} \log ^{3} x}{Q^{1 / 2}}\right)
\end{aligned}
$$

because of (92) again.

Taken all together, (90), (91), (96), (102), and (65) imply that expression (88) ignores the effect of a term

$$
\begin{aligned}
O\left(\frac{R x \log x}{Q}\right)+O( & \left.R x \log ^{4} x\right) \\
& +O\left(\frac{R^{3 / 2} x \log ^{4} x}{Q^{1 / 2}}\right)+O\left(\frac{R^{1 / 2} x^{3 / 2} \log ^{4} x}{Q^{1 / 2}}\right) \\
= & O\left(R x \log ^{4} x\right)+O\left(\frac{R^{3 / 2} x \log ^{4} x}{Q^{1 / 2}}\right)
\end{aligned}
$$

that emanates from $J_{1}^{*}(x, Q)$.

$\left({ }^{3}\right)$ Since, ultimately, $R$ will be chosen to exceed $x^{1 / 2}$, it may be wondered why this stipulation is not made now in order to simplify the calculations. The reason lies in our intention to perform an analogous calculation later on which the quantity corresponding to $R$ may be considerably smaller than $R$. 
Flowing as it does from the replacement for $p \nmid l$ of the remainder term $O(R)$ in $(75)$ by the expression

$$
\begin{aligned}
\sum_{\substack{d \leq R \\
(d, l)=1}} V(R) \lambda_{d}\left\{\psi^{-}\right. & \left.\left(\frac{p-l Q}{l d}-\frac{p \bar{d}}{l}\right)-\psi\left(-\frac{p \bar{d}}{l}\right)\right\} \\
& =\sum_{\substack{d \leq R \\
(d, l)=1}} V(R) \lambda_{d}\left\{\psi^{-}\left(-\frac{Q}{d}+\frac{p \bar{l}}{d}\right)-\psi\left(-\frac{p \bar{d}}{l}\right)\right\},
\end{aligned}
$$

the comparable analysis bound up with $J_{1}^{\dagger}(x, Q)$ in $(74)$ is very similar to what has gone before and results in there being another indirect effect on $S(x ; Q)$ of a contribution from $J_{1}^{\dagger}(x, Q)$ like (103) above.

The requisite improvement in the remainder term in the formula (79) for $J_{2}(x, Q)$ is derived by following the previous methods, although an extra entanglement is introduced because $\Lambda_{R}(n)$ is not necessarily non-negative and need not be zero when $(n, l)>1$ and $n>l$. Starting much as before, we employ (25) in Lemma 1 to state the remainder term in (79) as

$$
\begin{aligned}
& \sum_{l<x / Q} \sum_{m<x-l Q} \Lambda_{R}(m) \sum_{\eta \mid(m, l)} \sum_{\substack{d^{\prime} \leq \eta / R \\
\left(d^{\prime}, l^{\prime}\right)=1}} V(R) \lambda_{d^{\prime} \eta} \\
& \times\left\{\psi\left(\frac{x}{\eta l^{\prime} d^{\prime}}-\frac{(m / \eta) \overline{d^{\prime}}}{l^{\prime}}\right)-\psi\left(\frac{m+l Q}{\eta l^{\prime} d^{\prime}}-\frac{(m / \eta) \bar{d}^{\prime}}{l^{\prime}}\right)\right\}
\end{aligned}
$$

where $l^{\prime}$ denotes $l / \eta$. Next, since the condition $\eta \mid(m, l)$ is equivalent to the simultaneous congruences $m \equiv l \equiv 0, \bmod \eta$, this expression is seen to equal

$$
\begin{aligned}
& \sum_{\eta<x / Q} \sum_{l^{\prime}<x /(Q \eta)} \sum_{\substack{d^{\prime} \leq R / \eta \\
\left(d^{\prime}, l^{\prime}\right)=1}} V(R) \lambda_{d^{\prime} \eta} \\
& \times \sum_{m^{\prime}<\left(x-\eta l^{\prime} Q\right) / \eta} \Lambda_{R}\left(\eta m^{\prime}\right)\left\{\psi\left(\frac{x}{\eta l^{\prime} d^{\prime}}-\frac{m^{\prime} \bar{d}^{\prime}}{l^{\prime}}\right)-\psi\left(\frac{Q}{d^{\prime}}+\frac{m^{\prime} \bar{l}^{\prime}}{d^{\prime}}\right)\right\}
\end{aligned}
$$

through the use of the congruence $d^{\prime} \bar{d}^{\prime}+l^{\prime} \bar{l}^{\prime} \equiv 1, \bmod d^{\prime} l^{\prime}$, the innermost sum being

$$
\begin{aligned}
O\left(\frac{1}{N} \sum_{m^{\prime}<\left(x-\eta l^{\prime} Q\right) / \eta}\left|\Lambda_{R}\left(\eta m^{\prime}\right)\right|\right) \\
+O\left(\sum_{0<h \leq N} \frac{1}{h}\left|\sum_{m^{\prime}<\left(x-\eta l^{\prime} Q\right) / \eta} \Lambda_{R}\left(\eta m^{\prime}\right) e^{2 \pi i h \bar{d}^{\prime} m^{\prime} / l^{\prime}}\right|\right) \\
+O\left(\sum_{0<h \leq N} \frac{1}{h}\left|\sum_{m^{\prime}<\left(x-\eta l^{\prime} Q\right) / \eta}\right| \Lambda_{R}\left(\eta m^{\prime}\right)\left|e^{2 \pi i h \bar{d}^{\prime} m^{\prime} / l^{\prime}}\right|\right)
\end{aligned}
$$




$$
\begin{aligned}
& +O\left(\sum_{0<h \leq N} \frac{1}{h}\left|\sum_{m^{\prime}<\left(x-\eta l^{\prime} Q\right) / \eta} \Lambda_{R}\left(\eta m^{\prime}\right) e^{2 \pi i h \bar{l}^{\prime} m^{\prime} / d^{\prime}}\right|\right) \\
& +O\left(\sum_{0<h \leq N} \frac{1}{h}\left|\sum_{m^{\prime}<\left(x-\eta l^{\prime} Q\right) / \eta}\right| \Lambda_{R}\left(\eta m^{\prime}\right)\left|e^{2 \pi i h \bar{l}^{\prime} m^{\prime} / d^{\prime}}\right|\right)
\end{aligned}
$$

by Lemma 4 . The absence or presence of the modulus signs around $\Lambda_{R}\left(\eta m^{\prime}\right)$ in these exponential sums is irrelevant to our estimations of the sums obtained by replacing the innermost sum in (104) in turn by each of the last four constituents in (105), whence it is enough for us to consider the sums

$$
\begin{gathered}
\text { (106) } \log x \sum_{\substack{0<h \leq N \\
\eta<x / Q}} \frac{1}{h} \sum_{l^{\prime}<x /(Q \eta)} \sum_{\substack{d^{\prime} \leq R / \eta \\
\left(d^{\prime}, l^{\prime}\right)=1}}\left|\sum_{m^{\prime}<\left(x-\eta l^{\prime} Q\right) / \eta} \Lambda_{R}\left(\eta m^{\prime}\right) e^{2 \pi i h \bar{d}^{\prime} m^{\prime} / l^{\prime}}\right| \\
=\log x \sum_{\substack{0<h \leq N \\
\eta<x / Q}} \frac{1}{h} \Sigma_{h, 1}^{(\eta)}=\log x J_{2}^{\mathrm{I}}(x, Q), \quad \text { say, }
\end{gathered}
$$

and

$$
\begin{gathered}
\log x \sum_{\substack{0<h \leq N \\
\eta<x / Q}} \frac{1}{h} \sum_{\substack{d^{\prime} \leq R / \eta \\
l^{\prime}<x /(Q \eta) \\
\left(l^{\prime}, d^{\prime}\right)=1}}\left|\sum_{m^{\prime}<\left(x-\eta l^{\prime} Q\right) / \eta} \Lambda_{R}\left(\eta m^{\prime}\right) e^{2 \pi i h \bar{l}^{\prime} m^{\prime} / d^{\prime}}\right| \\
=\log x \sum_{\substack{0<h \leq N \\
\eta<x / Q}} \frac{1}{h} \Sigma_{h, 2}^{(\eta)}=\log x J_{2}^{\mathrm{II}}(x, Q), \quad \text { say, }
\end{gathered}
$$

together with the sum

$$
\frac{\log x}{N} \sum_{\eta<x / Q} \sum_{\substack{l^{\prime}<x /(Q \eta) \\ d^{\prime} \leq R / \eta}} \sum_{m^{\prime} \leq x / \eta}\left|\Lambda_{R}\left(\eta m^{\prime}\right)\right|
$$

that is

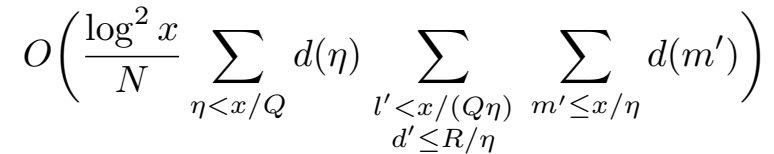

$$
\begin{aligned}
& =O\left(\frac{x^{2} R \log ^{3} x}{Q N} \sum_{\eta<x / Q} \frac{d(\eta)}{\eta^{3}}\right)=O\left(\frac{x^{2} R \log ^{3} x}{Q N}\right)=O(1)
\end{aligned}
$$

by (80) and (92).

The sums appearing in (106) and (107) are, respectively, very similar to those in (91) earlier on, save for the presence of the extra parameter $\eta$ and 
the replacement of the prime number function in the innermost sums by

$$
\Lambda_{R}\left(\eta m^{\prime}\right)=O\left\{\log x d(\eta) d\left(m^{\prime}\right)\right\} .
$$

Thus, on comparing $\Sigma_{h, 1}^{(\eta)}$ with the representation of $\Sigma_{h, 1}$ on the first line of (93), we see we can assess it exactly as the latter sum by allowing $\left({ }^{4}\right) x / \eta$ and $R / \eta$ to assume the rôles of $x$ and $R$ provided that we realize that the large sieve inequality will entail the manifestation of the sum

$$
\sum_{m^{\prime} \leq x / \eta} \Lambda^{2}\left(\eta m^{\prime}\right)=O\left(d^{2}(\eta) \log ^{2} x \sum_{m^{\prime} \leq x / \eta} d^{2}\left(m^{\prime}\right)\right)=O\left(\frac{d^{2}(\eta) x \log ^{5} x}{\eta}\right)
$$

instead of $\sum_{p \leq x} \log ^{2} p$. Since $R / \eta>(x / \eta) / Q$ by (65), the upshot is that we gain the estimate

$$
\Sigma_{h, 1}^{(\eta)}=O\left(\frac{d(\eta) d(h) R x \log ^{3} x}{\eta^{2}}\right)
$$

that is parallel to (95), whereupon we deduce from (106) that

$$
J_{2}^{\mathrm{I}}(x, Q)=O\left(R x \log ^{3} x \sum_{\substack{0<h \leq N \\ \eta<x / Q}} \frac{d(\eta) d(h)}{\eta^{2} h}\right)=O\left(R x \log ^{5} x\right) .
$$

Similarly, following the treatment of $J_{1}^{(2)}(x, Q)$, we derive the analogue

$$
\Sigma_{h, 2}^{(\eta)}=O\left(\frac{d(\eta) d(h) R^{3 / 2} x \log ^{3} x}{Q^{1 / 2} \eta^{5 / 2}}\right)+O\left(\frac{d(\eta) d(h) R^{1 / 2} x^{3 / 2} \log ^{3} x}{Q^{1 / 2} \eta^{2}}\right)
$$

of (101), whence on summation over $h$ and $\eta$ we conclude that

$$
J_{2}^{\mathrm{II}}(x, Q)=O\left(\frac{R^{3 / 2} x \log ^{5} x}{Q^{1 / 2}}\right)+O\left(\frac{R^{1 / 2} x^{3 / 2} \log ^{5} x}{Q^{1 / 2}}\right)
$$

after taking (92) into account.

The other relevant sums analogous to those in (106) and (107) are estimated in identical manner. Therefore, with (109), (110), and two similar majorizations, we deduce from (105) and (108) that the segment (88) also ignores the influence of a term

$$
\begin{array}{r}
O\left(R x \log ^{6} x\right)+O\left(\frac{R^{3 / 2} x \log ^{6} x}{Q^{1 / 2}}\right)+O\left(\frac{R^{1 / 2} x^{3 / 2} \log ^{6} x}{Q^{1 / 2}}\right) \\
=O\left(R x \log ^{6} x\right)+O\left(\frac{R^{3 / 2} x \log ^{6} x}{Q^{1 / 2}}\right)
\end{array}
$$

that proceeds from $J_{2}(x, Q)$. With this estimate, we have completed our reassessment of the appropriate remainder terms in the analysis by means of the second form of Lemma 1 and inequalities of large sieve type.

$\left({ }^{4}\right)$ Recall footnote $\left({ }^{3}\right)$. 
8. The conditional theorem. All that is left to be done is to combine our conclusion in Section 6 with our assessment of the effect of the work of Section 7 on $J_{1}\left(x ; Q_{1}, Q_{2}\right)$ and $J_{2}\left(x ; Q_{1}, Q_{2}\right)$. Recalling at once (88), (67), and the definitions (73) and (74), we see immediately from (103), its analogue for $J_{1}^{\dagger}(x, Q)$, and (111) that

$$
\begin{aligned}
& S\left(x, Q_{2}\right) \\
& \geq Q_{2} x \log \frac{R Q_{2}}{x}+O\left(Q_{2} x \log ^{1 / 2} x\right)+O\left(R x \log ^{6} x\right)+O\left(\frac{R^{3 / 2} x \log ^{6} x}{Q_{1}^{1 / 2}}\right) \\
& \geq Q_{2} x \log \frac{R Q_{2}}{x}+O\left(Q_{2} x \log ^{1 / 2} x\right)+O\left(R x \log ^{6} x\right)+O\left(\frac{R^{3 / 2} x \log ^{13 / 2} x}{Q_{2}^{1 / 2}}\right)
\end{aligned}
$$

on the strength of (56). In this, it is easily confirmed that the last two remainder terms can be absorbed by the first if $R \leq Q_{2} \log ^{-11 / 2} x$, which condition is not inconsistent with the requirement $R>x / Q_{1}$ in (60) because of (57). Hence, in the case where $Q_{2}$ satisfies both (57) and

$$
Q_{2} \leq x \exp \left(-\log ^{3 / 5} x\right)
$$

we deduce that

$$
S\left(x, Q_{2}\right) \geq Q_{2} x \log \left(\frac{Q_{2}^{2}}{x \log ^{11 / 2} x}\right)+O\left(Q_{2} x \log ^{1 / 2} x\right)
$$

by setting $R=Q_{2} \log ^{-11 / 2} x$, a comparable inequality being obtained when (112) fails by the choice $R=x \exp \left(-2 \log ^{3 / 5} x\right)$. Changing $Q_{2}$ into $Q$, we thus deduce

TheOREM 2. Suppose that the Riemann zeta function $\zeta(s)$ has no zeros with real part exceeding $3 / 4$. Then, for any real number $\alpha$ (possibly depending on $x$ ) between $1 / 2+\varepsilon_{1}$ and 1 and $Q=x^{\alpha}$, we have

$$
\sum_{k \leq Q} \sum_{\substack{0<a \leq k \\(a, k)=1}} E^{2}(x ; a, k)>\left(2-1 / \alpha-\varepsilon_{2}\right) Q x \log Q \quad\left(x>x_{0}\left(\varepsilon_{1}, \varepsilon_{2}\right)\right)
$$

in the notation of Theorem 1.

\section{References}

[1] J. B. Friedlander and D. A. Goldston, Variance of distribution of primes in residue classes, Quart. J. Math. Oxford Ser. (2) 47 (1996), 313-336.

[2] D. A. Goldston, A lower bound for the second moment of primes in short intervals, Exposition. Math. 13 (1995), 366-376.

[3] S. Graham, An asymptotic estimate related to Selberg's sieve, J. Number Theory 10 (1978), 83-94. 
[4] C. Hooley, Application of Sieve Methods to the Theory of Numbers, Cambridge Univ. Press, Cambridge, 1976.

[5] - On the Barban-Davenport-Halberstam theorem: I, J. Reine Angew. Math. 274/ 275 (1975), 206-223.

[6] - On the Barban-Davenport-Halberstam theorem: II, J. London Math. Soc. (2) 9 (1975), 625-636.

[7] -, On the Barban-Davenport-Halberstam theorem: XII, in: Number Theory in Progress (Zakopane, 1997), Vol. II, de Gruyter, 1999, 893-910.

[8] H. Q. Liu, Lower bounds for sums of Barban-Davenport-Halberstam type (supplement), Manuscripta Math. 87 (1995), 159-166.

[9] H. L. Montgomery, Maximal variants of the large sieve, J. Fac. Sci. Univ. Tokyo Sect. 1A 28 (1982), 805-812.

[10] E. C. Titchmarsh, The Theory of the Riemann Zeta-Function, Clarendon Press, Oxford, 1951.

[11] J. Vaaler, Some extremal functions in Fourier analysis, Bull. Amer. Math. Soc. (N.S.) 12 (1985), 183-216.

School of Mathematics

University of Wales

Senghennydd Road

Cardiff CF2 4YH, U.K. 